NBER WORKING PAPER SERIES

\title{
LEARNING FROM PRICES: \\ PUBLIC COMMUNICATION AND WELFARE
}

\author{
Manuel Amador \\ Pierre-Olivier Weill \\ Working Paper 14255 \\ http://www.nber.org/papers/w14255
NATIONAL BUREAU OF ECONOMIC RESEARCH
1050 Massachusetts Avenue
Cambridge, MA 02138

August 2008

We'd like to thank, for fruitful discussions and suggestions, George-Marios Angeletos, Andy Atkeson, Gadi Barlevy, Doireann Fitzgerald, Christian Hellwig, Narayana Kocherlakota, Nir Jaimovich, Kei Kawakami, Pat Kehoe, Ivan Werning, Randy Wright; and seminar participants at the Federal Reserve Bank of Cleveland Summer Workshops in Money, Banking and Payments, Stanford Junior Faculty Lunch, University of Pennsylvania, the Federal Reserve Bank of Minneapolis, MIT, NBER EFG Meeting, Queens University, Universite de Montreal, Washington University at St. Louis, UC Irvine, Rice University, Cal Poly, Paris X Nanterre and Paris School of Economics. We thank Marcello Miccoli for research assistance. All errors are ours. The views expressed herein are those of the author(s) and do not necessarily reflect the views of the National Bureau of Economic Research.

NBER working papers are circulated for discussion and comment purposes. They have not been peerreviewed or been subject to the review by the NBER Board of Directors that accompanies official NBER publications.

(C) 2008 by Manuel Amador and Pierre-Olivier Weill. All rights reserved. Short sections of text, not to exceed two paragraphs, may be quoted without explicit permission provided that full credit, including (c) notice, is given to the source. 
Learning from Prices: Public Communication and Welfare

Manuel Amador and Pierre-Olivier Weill

NBER Working Paper No. 14255

August 2008

JEL No. D83,E40,E58,E61

\begin{abstract}
We study the effect of releasing public information about productivity or monetary shocks when agents learn from nominal prices. While public releases have the benefit of providing new information, they can have the cost of reducing the informational efficiency of the price system. We show that, when agents have private information about monetary shocks, the cost can dominate, in that public releases increase uncertainty about fundamentals. In some cases, public releases can create or eliminate multiple equilibria. Our results are robust to adding velocity shocks, imperfectly observable prices, large idiosyncratic shocks, and introducing a bond market.
\end{abstract}

Manuel Amador

Stanford University

Department of Economics

Landau Economics Building

579 Serra Mall, Room 330

Stanford, CA 94305-6072

and NBER

amador@stanford.edu

Pierre-Olivier Weill

Department of Economics

University of California, Los Angeles

Bunche Hall 8283

Los Angeles, CA 90095

and NBER

poweill@econ.ucla.edu 


\section{Introduction}

Economic statistics are noisy. For example, the first estimates of GDP in the U.S., published by the Bureau of Economic Analysis, are very imprecise. Only half of the data required to compute it, is known completely at the time of the first release; and the original numbers are subsequently subject to significant revisions. ${ }^{1}$ Some economic statistics are even considered too noisy to be published: a recent example is the monetary aggregate M3 that the Federal Reserve Board stopped reporting in March 2006.

Should one be concerned that releasing noisy statistics may create confusion and lead the private sector to act on incorrect information? At first pass, the notion that more public information leads to more uncertainty and therefore worse decisions is unwarranted. Indeed, if we treat other sources of information as exogenous, a rational Bayesian decision maker will always be better informed after having observed a public signal, however noisy. But, in reality, not all sources of information are exogenous: households and firms learn through their interactions in markets and from observing variables such as prices. Our contribution in this paper is to show that when the information structure of an economy is endogenous, the release of public signals about aggregate fundamentals can actually lead to more confusion and greater uncertainty than no release at all. Moreover, public releases can create multiple Pareto-ranked equilibria, and consequently even modest announcements can result in large changes in equilibrium outcomes and uncertainty.

We provide a full characterization of the effects of public information releases in a monetary model inspired by that of Lucas (1972). In our cash-in-advance economy, households are uncertain about two aggregate disturbances, a monetary shock and a productivity shock. Households inhabit different locations and are asymmetrically informed regarding the two aggregate shocks. This dispersed private information about shocks is aggregated through the publicly observable equilibrium nominal prices prevailing every location. The nominal prices are affected not only by the underlying productivity shock but also by the unknown monetary shock, and, for that reason, households care indirectly about the monetary shock: knowledge about it helps them extract productivity information from nominal prices.

Our results are based on the following two observations. The first is that publicly observable prices not only generate new public information about productivity, they also generate

\footnotetext{
${ }^{1}$ See, for instance, the article "Why America's advance GDP figures do not paint the whole picture" published by The Economist on January $31^{\text {st }}, 2008$.
} 
new private information. This is because households extract productivity information from nominal prices by filtering out the monetary shock in two ways. On the one hand, they use their public knowledge about the monetary shock, which effectively generates an endogenous public signal about productivity. On the other hand, they use their private knowledge about the monetary shock, which generates an endogenous private signal about productivity.

The crucial decision for a household is choosing how much weight to give to its private versus its public information. Our second observation concerns the relationship between this optimal weighting decision and the two endogenous signals: the endogenous private signal tends to make households' weighting decisions strategic complements, while the endogenous public signal tends to make their weighting decisions strategic substitutes. The intuition for this second observation is the following. When other households put more weight on their private information, the two endogenous signals generated by prices become more informative about productivity, as more private information gets fed into the prices. Since the endogenous private signal is now more informative, an individual household will find it optimal to put more weight on it, a force for complementarity. At the same time, the endogenous public signal has become also more informative, and a household will then put more weight on it, and less weight on the private ones, a force for substitutability.

What is, then, the effect of releasing partial information about the monetary shock, the productivity shock, or both at the same time? As stated at the beginning, everything else equal, such releases have a direct beneficial effect of providing new information. There is, however, a countervailing equilibrium effect: households put more weight on the newly released public information and less on their private information. This change in behavior tends to reduce the endogenous informational content of prices. The strategic complementarities play the crucial role of amplifying this initial negative effect: households put less weight on the endogenous private signal, making prices less informative, prompting households to put even less weight on the endogenous private signal, making prices even less informative, and so on. In fact, due to this amplification mechanism, in equilibrium the negative effect can dominate the positive effect, increasing households' uncertainty and reducing welfare. Such amplification is necessary for the result: in its absence we show that public information is always beneficial.

Because of the strategic complementarities created by the endogenous private signal, our model can feature multiple Pareto-ranked equilibria differing in the degree of informativeness 
of nominal prices. For instance, in the high-informativeness, high-welfare equilibrium, other households put a large weight on their private information. The endogenous private signal generated by prices is thus more informative, making it optimal for an individual household to put a large weight on its private information. We also uncover another effect of public announcements: a release of sufficiently precise information about either the productivity or the monetary shock can guarantee a unique equilibrium. However, a mild release can, instead, generate multiplicity.

In our basic model we do not take a stand on the sources of the monetary shock and the reasons why the policy maker does not posses complete information about it. Subsection 5.1 addresses this question explicitly in an extension of the model with two different monetary shocks. The first shock is a change in a narrow monetary aggregate, which is assumed to perfectly observed by a Central Bank. The second shock is an aggregate velocity shock, representing changes in general credit conditions in the economy that are assumed to be imperfectly observed by a Central Bank and not known to the households. We also allow for a public noise component in the observation of nominal prices. We then proceed to show that the results from the basic model generalize to this multidimensional case, and obtain a general characterization of whether public releases are welfare reducing or not.

One may argue that the introduction of a financial market that could aggregate information might eliminate the non-fully revealing equilibrium that we obtain, which is necessary for the negative welfare results. In the subsection 5.2, we show that the basic model is robust to the introduction of a financial market where a nominal bond is traded.

There is a recent and influential literature documenting that, at the firm-level, prices and quantities are an order of magnitude more volatile than their corresponding aggregate (see, among others, Bils and Klenow, 2004). As such, a possible criticism of our analysis is that the economic agents are not too concerned about forecasting underlying macroeconomic shocks, but instead they are mainly responding to idiosyncratic shocks. We discuss in Subsection 5.3 that those criticisms are unwarranted. We present a simple modification of our baseline model with idiosyncratic productivity shocks. Although agents face arbitrarily volatile idiosyncratic shocks, social welfare is still driven by their uncertainty about the macroeconomic shocks: that is, the component that is affected by public announcements and the publication of economic statistics. 


\section{Literature Review}

Relative to the vast existing literature on public communication in macroeconomics, our main contribution is to show formally that more public information can lead to an overall increase of uncertainty about fundamentals. Although this effect has been conjectured before, it is not present in previous models of welfare reducing public information.

The basis of our analysis is a learning externality: agents respond to new public information by relying less on their own private information, which can reduce the informational content of prices. This externality has been noted and studied before, and our paper builds on the work of Vives (1993), Morris and Shin (2005), and Amato and Shin (2006). In these papers, however, the net effect of public information remains positive: it reduces agents uncertainty about fundamentals. Relative to this literature, our contribution is threefold. First, we uncover an amplification mechanism for the learning externality to generate, on its own, an increase in uncertainty about fundamentals: we show that some of the endogenous information gathered by agents has to be private. Second, we formalize the argument in a familiar macroeconomic model which allows for a micro-founded welfare analysis. And finally, we show that this private information gathering arises naturally from the public observation of prices. This last step is important since prices arguably constitute the most important channel of information aggregation and are publicly observed.

A variant of the basic result also appears in our contemporaneous paper, Amador and Weill (2006). However, the focus of Amador and Weill (2006) is on transitional and long-run information dynamics in an abstract learning model; which lacking micro-foundations, is not well suited for welfare analysis.

We have chosen to develop our theoretical argument with a model in the spirit of Lucas (1972). This framework is familiar to most macro-economists and is a canonical example of imperfect information. However, most of our results are easily generalizable to other settings, as long as agents are learning from an endogenous source of information, such as in the noisy rational expectations models of Grossman (1975) and Hellwig (1980). One advantage of our flexible price setup is to clearly separate our mechanism from the ones based on pricing complementarities, which have been the center of the macroeconomic literature building on Morris and Shin (2002) (as described in the next paragraph). That said, one could make the model more realistic by adding real rigidities or other sources of complementarities, but at the cost of making the results less transparent. 
In a recent influential work, Morris and Shin (2002) have proposed a different, coordinationbased mechanism of the welfare cost of public information releases. Agents desire to achieve coordination in their actions, but such behavior is socially undesirable. For that reason a public signal, by allowing agents to coordinate their actions, can be socially harmful, even though it reduces agents' uncertainty about fundamentals. Whether such a trade-off is relevant or not for policy (and in particular monetary policy) has been the subject of much discussion. It is now known that in the coordination-based neo-keynesian models of Hellwig (2005), Roca (2006), and Lorenzoni (2007), there is no trade-off: releases of public information are socially beneficial. More generally, Angeletos and Pavan (2007) have found the conditions on linear-quadratic preferences under which public information releases are socially harmful, and have shown that the negative welfare results are very sensitive to the preference structure. Similarly to Morris and Shin (2002), our results are driven by the impact of public information on agents' relative weighting of private versus public information. Differently, in our model, agents do not suffer from a socially harmful desire to coordinate: public information releases are welfare reducing because they increase agents' uncertainty about fundamentals. Our model also has the positive implication that the publication of economic statistics can result in less accurate forecasts, a prediction which is amenable to empirical work. The virtue of this prediction is its simplicity: testing it does not require the econometrician to make additional assumptions on preferences parameters, or on the social value of coordination.

Lastly, several authors have studied the interactions of public communication with public policy (see Moscarini, 2007; Atkeson et al., 2007; Eusepi and Preston, 2007; Taub, 1997). Our approach here applies to the publication of economic statistics as well as communication of economic outlook, taking all other aspects of public policy as given.

The rest of the paper is organized as follows. Section 2 starts by presenting the basic model set up, and proceeds by defining and characterizing its linear equilibria. The second main result on welfare effects is analyzed in Section 4. Section 5 studies three extensions. Subsection 5.1 introduces velocity shocks and imperfect observability of the price level, and studies optimal announcements of narrow versus broad monetary aggregates. We obtain a simple characterization of whether a release of marginal public information about any shock is welfare reducing: it occurs if the ratio of public to private knowledge about that particular shock is sufficiently small. Subsection 5.2 shows that the opening of a bond market 
that could aggregate more information does not change the results. Subsection 5.3 shows that our results are robust to the introduction of idiosyncratic productivity shocks. Section 6 concludes. All omitted proofs are in the appendix.

\section{The Model}

We consider a standard cash-in-advance model modified to allow for three features of interest. First, we introduce a real shock that affects the cost of producing that, initially, is imperfectly and differentially known by households. Second, we allow for differential information among households regarding the monetary shock (such heterogeneity is naturally generated by idiosyncratic initial money holdings). Finally, as in Townsend (1983), even though there is no trade across locations, every household observes the economy-wide distribution of nominal prices and learns from them.

\section{Preferences and Technology}

Time is discrete. Although the model is essentially static, we let time be infinite so that money is valued. The economy is composed of a $[0,1]$-continuum of locations. In each location there are competitive firms operating a linear technology, transforming one unit of labor into one unit of the consumption good. Firms hire labor in a competitive local labor market and sell their output in a competitive local goods market. Because labor and goods are immobile, the relative wage in terms of the consumption good is unity in all locations.

At each time $t \in\{1,2, \ldots\}$ in location $i \in[0,1]$ a representative household chooses its effort supply, $L_{i t}$, consumption, $C_{i t}$, and money balance, $M_{i t}^{d}$, in order to maximize

$$
\mathbb{E}_{i 1}\left[\sum_{t=1}^{\infty} \beta^{t-1}\left(\log \left(C_{i t}\right)-\Theta L_{i t}\right)\right]
$$

where $\Theta$ represent an aggregate permanent effort cost and subject to sequence of budget and cash-in-advance (CIA) constraints,

$$
\begin{aligned}
C_{i t}+\frac{M_{i t}^{d}}{P_{i t}} & \leq L_{i t}+\frac{M_{i t-1}^{d}}{P_{i t}} \\
C_{i t} & \leq \frac{M_{i t-1}^{d}}{P_{i t}}
\end{aligned}
$$


where $P_{i t}$ denotes the nominal price level in location $i$ at time $t$. The initial money balance of the representative household of location $i$ is $M_{i 0}^{d}=M_{i}$.

\section{Exogenous Information about Money}

At time $t=1$, an aggregate monetary shock causes households to be uncertain about the aggregate money supply: they share the common prior that the logarithm of the aggregate money supply is $m+\bar{m}$, where $m$ is a normally distributed monetary shock with mean zero, precision $\Psi_{m}$, and $\bar{m}$ is some constant known to everyone.

In practice, how could households be uncertain about the money supply? First, although the Federal Reserve Board publishes weekly data on money aggregates M1 and M2, the estimates are subsequently revised as depository institutions either report new data or revise the data they previously reported. ${ }^{2}$ In addition, one may argue that even error-free measures of M1 and M2 remain noisy estimates of the "true" measure of aggregate liquidity that enters the quantity equation and directly influences the price level. This measure of aggregate liquidity may include some less liquid assets omitted in M1 and M2 and could be influenced by unobserved velocity shocks. We formalize this argument in Section 5.1 by adding velocity shocks to the model.

The initial money endowment of the representative household of location $i \in[0,1]$ is

$$
\log M_{i} \equiv \hat{m}_{i}=m+\varepsilon_{m i}
$$

where $\varepsilon_{m i}$ is normally distributed across locations with mean zero and precision $\psi_{m}$. Thus, the logarithm of the aggregate money supply is $\log M \equiv m+\left(2 \psi_{m}\right)^{-1}$. $^{3}$

Note that the initial money endowment constitutes private information about the aggregate money supply. This initial private information will be the key driver of our result. ${ }^{4}$

\footnotetext{
${ }^{2}$ See the December 2006 "Performance Evaluation of the Statistical Release about Money Stock Measures" on the Federal Reserve Board website: http://www .federalreserve.gov/releases/h6/perfeval2006.htm

${ }^{3}$ Hence, $\bar{m}=\left(2 \psi_{m}\right)^{-1}$. We could shift the mean of $\hat{m}_{i}$ up and down by any constant to obtain a different $\bar{m}$, without changing any of our results.

${ }^{4}$ In all what follows, "private information" refers to a piece of information that is known solely by households in a given location.
} 


\section{Exogenous Information about Productivity}

A key timing assumption of our model is that the cost of effort, $\Theta$, is unknown as of time $t=1$ but is revealed to everyone at $t=2$. The objective of this timing assumption is to introduce a risky investment in the model. Indeed, in the first period of our equilibrium, households will choose the amount of effort, $L_{i 1}$, to put in their work, but the return on their investment will have a random component, $-\Theta L_{i 1}$. While there are, of course, other and perhaps more standard models of risky investment, our timing assumption has the advantage of keeping the analysis tractable and transparent. We assume that all households share the common prior that $\log (\Theta) \equiv \theta$ is normally distributed with a mean of zero and precision $\Psi_{\theta}$, and is independent of the aggregate money supply. Households also observe a private signal about the effort cost:

$$
\hat{\theta}_{i}=\theta+\varepsilon_{\theta i}
$$

where $\varepsilon_{\theta i}$ is normally distributed with mean of zero and precision $\psi_{\theta}$.

We assume that the random variables $\theta, m, \varepsilon_{\theta i}$ and $\varepsilon_{m i}$, are all pairwise independent. ${ }^{5}$ In what follows, we will use uppercase $\Psi$ (lowercase $\psi$ ) to denote the precisions of public (private) information about aggregate variables. As is standard in the literature, we measure the amount of information in precision units. For instance, the amount of public information about money is measured by $\Psi_{m}$. The overall precision structure is summarized in Table 1. Importantly, public releases of exogenous information about $m$ and $\theta$ translate into increases in $\Psi_{m}$ and $\Psi_{\theta}{ }^{6}{ }^{6}$ Hence, in order to study the effect of public information releases, it suffices to conduct comparative static exercises with respect to the exogenous parameters $\Psi_{m}$ and $\Psi_{\theta}$.

\footnotetext{
${ }^{5}$ Sun (2006) provides the precise construction of a probability space where the exact law of large numbers holds for a continuum of pairwise independent random variables.

${ }^{6}$ For example, suppose households start with a common prior has precision $\bar{\Psi}_{\theta}$ and receive a public signal $\theta+u$, where $u$ is normally distributed and independent from everything else, with a mean of zero and a precision of $\Delta_{\theta}$. Then, the households' posterior precision is equal to $\bar{\Psi}_{\theta}+\Delta_{\theta}$, and releasing public information amounts to increasing $\Psi_{\theta}$. Importantly, in this comparative static exercise, one should keep constant the precision $\bar{\Psi}_{\theta}$ of the common prior: indeed, increasing $\bar{\Psi}_{\theta}$ at the same time would reduce fundamental volatility, which may have a confounding impact on ex ante welfare. The proof of Proposition 5 shows that we do not need, however, to be explicit about holding $\bar{\Psi}_{\theta}$ constant: welfare turns out to depend only on the posterior precision $\Psi_{\theta}=\bar{\Psi}_{\theta}+\Delta_{\theta}$ and on the prior mean of $\log \Theta$, which is equal to zero.
} 
Table 1: Precision structure.

\begin{tabular}{c|cc}
\hline \hline & Money supply: $m$ & Effort shock: $\theta$ \\
\hline Public Information & $\Psi_{m}$ & $\Psi_{\theta}$ \\
Private Information & $\psi_{m}$ & $\psi_{\theta}$ \\
\hline
\end{tabular}

\section{Endogenous Information from Nominal Prices}

The only way the locations are connected is informationally: households observe the distribution of nominal prices of the entire economy when making their labor supply and consumption decisions. Let us denote by $p_{t}$ the average logarithmic price across locations:

$$
p_{t}=\int p_{i t} d i
$$

As we will show later on, the average price level will become a sufficient statistic of the entire price distribution in equilibrium.

\section{Market Clearing Conditions}

We assume that goods and labor cannot flow across locations, so that the goods market must clear locally:

$$
C_{i t}=L_{i t}
$$

for all $i \in[0,1]$ and $t \in\{1,2, \ldots\}$. Also the money markets are in equilibrium: ${ }^{7}$

$$
M_{i t}^{d}=M_{i}
$$

\subsection{Equilibrium}

An equilibrium is made up of a sequence of distributions of consumption, labor and money holdings across locations, together with a distribution of prices in the economy such that, at each time,

(i) given the information conveyed by the distribution of prices in the economy and given the local price, households choose consumption, labor and money holdings to maximize

\footnotetext{
${ }^{7}$ We could instead impose an aggregate money market clearing condition: $\int M_{i t}^{d} d i=M$. Indeed, the fact that goods and factors are immobile will, by applying Walras' Law in each location, imply condition (8).
} 
their expected utility;

(ii) every local goods market clears.

Before characterizing an equilibrium formally, it is convenient to first analyze the household's problem.

\section{Solving the Household's Problem}

Consider the representative household of location $i \in[0,1]$ and let $\beta^{t-1} \lambda_{i t}$ and $\beta^{t-1} \mu_{i t}$ be the non-negative Lagrange multipliers of its budget constraint (2) and CIA constraint (3). Then, the first-order conditions for consumption, labor and money balances are

$$
\begin{aligned}
\frac{1}{C_{i t}} & =\lambda_{i t}+\mu_{i t} \\
\mathbb{E}_{i t}[\Theta] & =\lambda_{i t} \\
\frac{\lambda_{i t}}{P_{i t}} & =\beta \mathbb{E}_{i t}\left[\frac{\lambda_{i t+1}+\mu_{i t+1}}{P_{i t+1}}\right],
\end{aligned}
$$

where the expectation operator, $\mathbb{E}_{i t}[\cdot]$, is conditional on all the information available to household $i$ as of time $t$.

Anticipating that the CIA constraint binds at all times, that is $C_{i t}=M_{i t-1}^{d} / P_{i t}$, it follows that $\lambda_{i t}+\mu_{i t}=P_{i t} / M_{i t-1}^{d}$. In addition, plugging the goods market clearing condition (7) into the binding budget constraint (2), we obtain

$$
M_{i t}^{d}=M_{i t-1}^{d}
$$

implying that a household's money holdings must stay equal to its initial money endowment at each time, i.e. $M_{i t}^{d}=M_{i}$ (the money market is clearing). Now, together with equations (10) and (11), these manipulations show that ${ }^{8}$

$$
P_{i t}=\beta^{-1} M_{i} \mathbb{E}_{i t}[\Theta]
$$

Plugging this back into the binding CIA constraint, we obtain an inverse relationship between

\footnotetext{
${ }^{8}$ The price level in each location is uniquely determined by the expectations of $\Theta$ and the money holdings: there are not multiple monetary equilibria (a common occurrence in monetary models). This determinacy is due to our log preference specification.
} 
consumption and the expected effort cost,

$$
C_{i t}=\beta \mathbb{E}_{i t}[\Theta]^{-1}
$$

Plugging this into equation (9), we obtain that $\mathbb{E}_{i}[\Theta] / \beta=\lambda_{i t}+\mu_{i t}$. Together with (10), this implies that $\mu_{i t}=(1-\beta) \mathbb{E}_{i t}[\Theta] / \beta$ is strictly positive, confirming our guess that the CIA constraint is binding at all times.

Note that the households are concerned only with the cost parameter $\Theta$. Because the goods markets clear locally and the local money supply is constant and known, uncertainty about the aggregate money supply does not directly affect the households' problem. However, as seen in equation (12), nominal prices in the economy combine local expectations about the cost parameter and local money supplies. Thus, information about money will affect households' ability to extract information about $\Theta$ from observing the economy-wide prices.

\section{Equilibrium After the Second Period}

From period $t=2$ onwards, households know the exact realization of $\Theta$. Hence, we have from equation (12) that, in equilibrium

$$
\begin{aligned}
& P_{i t}=\beta^{-1} M_{i} \Theta \\
& C_{i t}=L_{i t}=\beta \Theta^{-1}
\end{aligned}
$$

for all $t \geq 2$. So quantities and prices are determined from $t=2$ onwards.

\section{Linear Equilibrium in the First Period}

As we proceed to study the competitive equilibrium in the first period, and given that the economy is stationary from $t=2$ onwards, we simplify notations by removing the time subscript from all first-period variables.

Borrowing from the literature on noisy rational expectations in financial markets (see, among many others, Grossman, 1975; Hellwig, 1980), we restrict ourselves to linear equilibria:

Definition 1 (Linear Equilibrium). A linear equilibrium is a cross-sectional distribution of nominal prices, $P_{i}$, consumption, $C_{i}$, effort supplies, $L_{i}$, and expectations about $\Theta, \mathbb{E}_{i}[\Theta]$, such that 
i) conditional on the realization of $(m, \theta)$, the distribution of prices is log-normal with constant dispersion and a mean parameter

$$
p=\alpha_{0}+\alpha_{1} \theta+\alpha_{2} m
$$

for some constants $\alpha_{0}, \alpha_{1}$ and $\alpha_{2}$;

ii) households' expectations are rational; that is, after observing their private signals $\left(\hat{m}_{i}, \hat{\theta}_{i}\right)$ and the distribution of nominal prices in the economy,

$$
\mathbb{E}_{i}[\Theta]=\mathbb{E}\left[\Theta \mid \hat{\theta}_{i}, \hat{m}_{i}, p\right]
$$

iii) households decisions are optimal and markets clear:

$$
\begin{aligned}
C_{i} & =L_{i}=\beta \mathbb{E}_{i}[\Theta]^{-1} \\
P_{i} & =\beta^{-1} M_{i} \mathbb{E}_{i}[\Theta] .
\end{aligned}
$$

To understand our rational expectations condition (15), note the following: even though households observe the entire cross-sectional distribution of nominal prices, it is sufficient for them to condition expectations with respect to only one moment of the distribution, the average price level $p$. Indeed, in the equilibria we consider, the distribution of prices in the economy is log-normal and, hence, is uniquely parameterized by its mean and its dispersion. Given the additional requirement in part (i) that the dispersion does not depend on the realization $(m, \theta)$, the mean parameter, $p$, conveys all the information embedded in the price distribution.

\section{Equilibrium Characterization}

In this section we proceed to characterize the set of equilibria.

\subsection{The Household's Information Updating Problem}

The analysis of the household's updating problem is based on the following insight: although prices are publicly observable, they also generate private information about productivity. 


\section{A Transformation of the Household's Information Set}

We start by noting that observing the average log-price, $p$, is informationally equivalent to a signal $\hat{z}$, such that

$$
\hat{z}=\theta+m / A
$$

where $A=\alpha_{1} / \alpha_{2}$, given that $\alpha_{0}, \alpha_{1}$ and $\alpha_{2}$ are constant. A simple transformation of households' information set implies:

Lemma 1. The joint observation of $\hat{\theta}_{i}=\theta+\varepsilon_{\theta i}, \hat{m}_{i}=m+\varepsilon_{m i}$ and $\hat{z}=\theta+m / A$ is equivalent to the joint observation of

$$
\begin{aligned}
\hat{\theta}_{i} & =\theta+\varepsilon_{\theta i} \\
\hat{z} & =\theta+m / A \\
\hat{z}_{i} \equiv \hat{z}-\hat{m}_{i} / A & =\theta-\varepsilon_{m i} / A .
\end{aligned}
$$

Proof. This follows immediately by replacing $\hat{m}_{i}$ by $\hat{z}_{i} \equiv \hat{z}-\hat{m}_{i} / A$ in a household's infor-

mation set, while keeping the two other observations, $\hat{\theta}_{i}$ and $\hat{z}$, the same.

Observing the price level, $p$, generates two independent signals about $\theta$. There is first a public signal of precision $A^{2} \Psi_{m}$, given by (20), which intuitively follows from extracting productivity information from the price level, based on the public information about the money supply. Second, the price also generates a private signal about $\theta$ of precision $A^{2} \psi_{m}$, given by (21), which follows from extracting productivity information from the price level, based on the private information, $\hat{m}_{i}$, about the money supply. The finding that the publicly observable price level also generates a private signal is the main insight of the lemma, and will be a key driver of our results.

\section{Information Updating}

Given the three signals of Lemma 1, a household proceeds to calculate its posterior beliefs about $\Theta$. In order to build intuition, we decompose the household's posterior-belief calculation in the following three steps.

First, conditional on its private information, $\hat{\theta}_{i}$ and $\hat{z}_{i}$, and given a fully diffuse prior, a household forms a private posterior belief that $\Theta$ is log-normally distributed with a mean 
and precision:

$$
\begin{aligned}
\mathbb{E}\left[\theta \mid \hat{\theta}_{i}, \hat{z}_{i}\right] & =\frac{\psi_{\theta}}{\psi_{\theta}+A^{2} \psi_{m}} \hat{\theta}_{i}+\frac{A^{2} \psi_{m}}{\psi_{\theta}+A^{2} \psi_{m}} \hat{z}_{i} \\
\psi_{\theta}^{\prime} & =\psi_{\theta}+A^{2} \psi_{m} .
\end{aligned}
$$

Equation (22) is the standard Bayesian updating formula for independent signals and normal distribution: the private posterior belief about $\theta$ is a convex sum of the two private signals, with convex weights reflecting the signals' relative precisions. The posterior private precision, $\psi_{\theta}^{\prime}$, is obtained by adding up the precisions of the two private signals.

Secondly, conditional on the public signal, $\hat{z}$, and given the common prior that $\Theta$ is $\log$ normally distributed with mean zero and precision $\Psi_{\theta}$, a household forms the public posterior belief that $\Theta$ is log-normally distributed with mean and precision

$$
\begin{aligned}
\mathbb{E}[\theta \mid \hat{z}] & =\frac{A^{2} \Psi_{m}}{\Psi_{\theta}+A^{2} \Psi_{m}} \hat{z} \\
\Psi_{\theta}^{\prime} & =\Psi_{\theta}+A^{2} \Psi_{m} .
\end{aligned}
$$

Finally, a household combines the private and the public belief together to form their fullinformation posterior belief. Namely, conditional on both the private and the public signals, a household's posterior beliefs is that $\Theta$ is log-normally distributed, with a mean given by

$$
\mathbb{E}_{i}[\theta]=a \mathbb{E}\left[\theta \mid \hat{\theta}_{i}, \hat{z}_{i}\right]+(1-a) \mathbb{E}[\theta \mid \hat{z}]
$$

which is a convex combination of the private posterior belief and the public posterior belief. The convex weight a reflects, as usual, the relative precision of the private and the public information:

$$
a=\frac{\psi_{\theta}^{\prime}}{\psi_{\theta}^{\prime}+\Psi_{\theta}^{\prime}}=\frac{\psi_{\theta}+A^{2} \psi_{m}}{\psi_{\theta}+A^{2} \psi_{m}+\Psi_{\theta}+A^{2} \Psi_{m}}
$$

The household's full-information posterior precision about $\Theta$ is equal to $\psi_{\theta}^{\prime}+\Psi_{\theta}^{\prime}$, the sum of the private and public precision. 


\subsection{Information Aggregation}

Having solved for the household's beliefs given the information structure, we proceed now to characterize the equilibrium distribution of prices. We verify our guess that the average log price is observationally equivalent to observing a signal $\hat{z}=\theta+m / A$. Importantly, we show that, in an equilibrium, the $A$ parameter must be equal to the weight $a$ that an individual household puts on its private information.

\section{Equilibrium Prices}

From the logarithms of (17) it follows that

$$
\log \left(P_{i}\right)=p_{i}=-\log \beta+\hat{m}_{i}+\mathbb{E}_{i}[\theta]+\frac{1}{2\left(\psi_{\theta}^{\prime}+\Psi_{\theta}^{\prime}\right)},
$$

where the last term appears because of Jensen's inequality. Taking the cross-sectional average, we obtain that the average log price is

$$
\begin{aligned}
p & =\int p_{i} d i=-\log \beta+\int \hat{m}_{i} d i+\int \mathbb{E}_{i}[\theta] d i+\frac{1}{2\left(\psi_{\theta}^{\prime}+\Psi_{\theta}^{\prime}\right)} \\
& =-\log \beta+m+a \int \mathbb{E}\left[\theta \mid \hat{\theta}_{i}, \hat{z}_{i}\right] d i+(1-a) \mathbb{E}[\theta \mid \hat{z}]+\frac{1}{2\left(\psi_{\theta}^{\prime}+\Psi_{\theta}^{\prime}\right)} \\
& =-\log \beta+m+a \theta+(1-a) \mathbb{E}[\theta \mid \hat{z}]+\frac{1}{2\left(\psi_{\theta}^{\prime}+\Psi_{\theta}^{\prime}\right)}
\end{aligned}
$$

The second line follows from substituting equation (26) into the first line. The third line, (29), follows because, by equation (22), the private beliefs $\mathbb{E}\left[\theta \mid \hat{\theta}_{i}, \hat{z}_{i}\right]$ are independent and centered around $\theta$. Now, given that the public belief $\mathbb{E}[\theta \mid \hat{z}]$ is known to everyone, the only part of the average log price that is unknown to households is $m+a \theta$. Hence, observing the average log price is equivalent to observing $m+a \theta$ or, after dividing by $a$, observing $\theta+m / a$. Comparing with our guess (18), we obtain the equilibrium condition

$$
A=a .
$$

Taken together, the best reply (27) and the above equilibrium condition (30) imply that $A$ must solve the fixed-point equation

$$
A=\frac{\psi_{\theta}+A^{2} \psi_{m}}{\psi_{\theta}+A^{2} \psi_{m}+\Psi_{\theta}+A^{2} \Psi_{m}} .
$$


The right hand side of this fixed-point equation is a continuous mapping from the real line into $[0,1]$. It follows, then:

Proposition 1. There exists at least one solution to the fixed-point equation (31). And there is a one-to-one correspondence between solutions of this equation and linear equilibria.

\subsection{Complementarities and Substitutabilities in Actions}

Before proceeding into the characterization of the equilibria, in this subsection we make an observation that is important for understanding our results: the endogenous private information generated by prices is shown to make households' actions strategic complements, while the endogenous public information is shown to make them strategic substitutes.

Recall that a household's posterior belief about $\theta$ can be written as the convex combination $a \mathbb{E}\left[\theta \mid \hat{\theta}_{i}, \hat{z}_{i}\right]+(1-a) \mathbb{E}[\theta \mid \hat{z}]$ of its private and public beliefs. The convex weight $a$ thus represents the weight a household puts on its private information. The individually optimal weight is given by the best-reply function (27), which can be written

$$
a=H\left(\psi_{\theta}^{\prime}, \Psi_{\theta}^{\prime}\right)=H\left(\psi_{\theta}+A^{2} \psi_{m}, \Psi_{\theta}+A^{2} \Psi_{m}\right)
$$

where $H\left(\psi_{\theta}^{\prime}, \Psi_{\theta}^{\prime}\right) \equiv \psi_{\theta}^{\prime} /\left(\psi_{\theta}^{\prime}+\Psi_{\theta}^{\prime}\right)$. Furthermore, in (32), $A$ can be interpreted as the weight chosen by all other households: the "aggregate weight."

When the aggregate weight $A$ increases, that is when other households rely more on their private information, prices aggregate more new private information. This increases both the precision $A^{2} \psi_{m}$ of the private signal and the precision $A^{2} \Psi_{m}$ of the public signal generated by prices. These increases have two opposite effects on the individually optimal weight, $a$.

The first effect, which follows because $\partial H / \partial \psi_{\theta}^{\prime}>0$, tends to increase $a$. Indeed, when $A$ goes up, the precision of the private signal generated by prices goes up, and an individual household's total private information, $\psi_{\theta}^{\prime}=\psi_{\theta}+A^{2} \psi_{m}$, increases. Holding public information $\Psi_{\theta}^{\prime}$ the same, an individual household will find it optimal to rely more on this improved private information, i.e., to increase $a$. Therefore, the endogenous private signal generated by prices tends to make households' weighting decisions strategic complements.

Because $\partial H / \partial \Psi_{\theta}^{\prime}<0$, there is also an opposite effect. When $A$ increases, the precision of the public signal generated by prices increases as well, and an individual household's total public information, $\Psi_{\theta}^{\prime}=\Psi_{\theta}+A^{2} \Psi_{m}$, increases. Holding private information $\psi_{\theta}^{\prime}$ constant, an 
individual household will find it optimal to rely more on this improved public information and less on its private information, i.e., to decrease $a$. Therefore, the endogenous public signal generated by price tends to make households' actions strategic substitutes.

Which of the two effect dominates depends on parameter values. To see this, note that $H\left(\psi_{\theta}^{\prime}, \Psi_{\theta}^{\prime}\right)=H\left(\psi_{\theta}^{\prime} / \Psi_{\theta}^{\prime}, 1\right)$ is homogenous of degree zero in $\left(\psi_{\theta}^{\prime}, \Psi_{\theta}^{\prime}\right)$. Since $H$ increases in its first argument, it follows that the best reply (32) is increasing in $A$ if and only if the ratio $\psi_{\theta}^{\prime} / \Psi_{\theta}^{\prime}$ is increasing in $A$, that is,

Lemma 2. The best reply function, $H$, increases in $A$ if and only if

$$
\frac{1}{\psi_{\theta}^{\prime}} \frac{\partial \psi_{\theta}^{\prime}}{\partial A}>\frac{1}{\Psi_{\theta}^{\prime}} \frac{\partial \Psi_{\theta}^{\prime}}{\partial A} \Longleftrightarrow \frac{\psi_{m}}{\Psi_{m}}>\frac{\psi_{\theta}}{\Psi_{\theta}}
$$

That is, if and only if, when $A$ increases, the total precision of private information grows faster than the total precision of public information.

Proof. Follows directly from taking derivatives and rearranging.

The lemma provides an intuitive condition for households' actions to be strategic complements. When $\psi_{m}$ is large or when $\Psi_{m}$ is small, the private channel generates proportionally more information than the public one. In this case, an increase in the aggregate weight, $A$, increases the precision of the private signal proportionally more than the public one, a force for complementarities. Also, when $\psi_{\theta}$ is small or $\Psi_{\theta}$ is large the public prior information before observing prices is more precise than the private: this implies that the posterior private precision, $\psi_{\theta}+A^{2} \psi_{m}$, is proportionally more affected by an increase in $A$ than the public posterior precision, $\Psi_{\theta}+A^{2} \Psi_{m}$. This is, again, a force for complementarities.

\subsection{Public Information and Multiplicity}

This subsection explains how the complementarities identified above may create multiple equilibria. We also explain the impact on multiplicity of changing the public information as given by $\left(\Psi_{m}, \Psi_{\theta}\right)$.

To that end, we start by fixing the private information parameters $\left(\psi_{m}, \psi_{\theta}\right)$ and we let $\mathcal{M}\left(\psi_{m}, \psi_{\theta}\right)$ be the set of $\left(\Psi_{m}, \Psi_{\theta}\right)$ such that there are multiple (more than one) equilibria. We start by three elementary properties: 
Proposition 2. The set function $\mathcal{M}\left(\psi_{m}, \psi_{\theta}\right)$ has the following properties: (i) it is empty if and only if $\psi_{m} / \psi_{\theta} \leq 27$, (ii) it is homogeneous of degree one, i.e., for all $\mu>0$, $\mathcal{M}\left(\mu \psi_{m}, \mu \psi_{\theta}\right)=\mu \mathcal{M}\left(\psi_{m}, \psi_{\theta}\right)$, and (iii) it is bounded.

The first point of the proposition simply says that, if $\psi_{m} / \psi_{\theta} \leq 27$, then there exists a unique equilibrium for any pair $\left(\Psi_{m}, \Psi_{\theta}\right)$, and otherwise, there exists multiple ones for some $\left(\Psi_{m}, \Psi_{\theta}\right)$. The second point of the proposition follows directly from the fact that the equilibrium equation (31) is homogenous of degree zero in the precision parameters: scaling all precision parameters $\left(\psi_{m}, \psi_{\theta}, \Psi_{m}, \Psi_{\theta}\right)$ up and down by the same constant won't change the set of equilibrium weights, $A$. This means that multiplicity does not depend on the absolute level of information, but on how the information is divided among the different sources. What does matter for multiplicity is the relative amount of public information, ( $\left.\Psi_{m}, \Psi_{\theta}\right)$, versus private information, $\left(\psi_{m}, \psi_{\theta}\right)$. Indeed, as shown in the third point of the proposition, for each $\left(\psi_{m}, \psi_{\theta}\right)$, the set $\mathcal{M}\left(\psi_{m}, \psi_{\theta}\right)$ is bounded; that is, holding $\left(\psi_{m}, \psi_{\theta}\right)$ fixed, if the amount $\left(\Psi_{m}, \Psi_{\theta}\right)$ of public information is sufficiently precise, there exists a unique equilibrium.

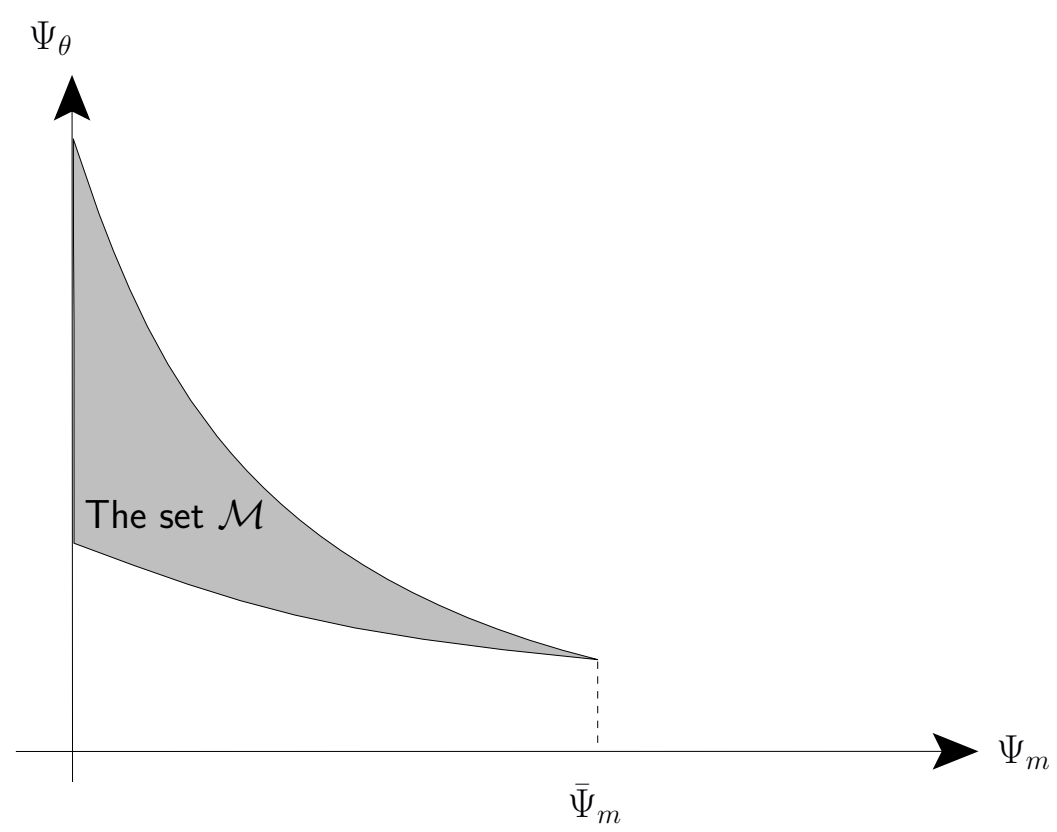

Figure 1: The set $\mathcal{M}$ and its boundaries.

To simplify notations, from now on we suppress the dependence of $\mathcal{M}$ on the private information vector $\left(\psi_{m}, \psi_{\theta}\right)$. 
Proposition 3. For all $\left(\psi_{m}, \psi_{\theta}\right)$ such that $\psi_{m} / \psi_{\theta}>27$, the set $\mathcal{M}$ is delimitated by continuous, strictly positive, strictly decreasing upper and lower boundaries

$$
\begin{aligned}
& U\left(\Psi_{m}\right)=\max \left\{\Psi_{\theta}:\left(\Psi_{\theta}, \Psi_{m}\right) \in \mathcal{M}\right\} \\
& L\left(\Psi_{m}\right)=\min \left\{\Psi_{\theta}:\left(\Psi_{\theta}, \Psi_{m}\right) \in \mathcal{M}\right\},
\end{aligned}
$$

defined for $0 \leq \Psi_{m}<\bar{\Psi}_{m} \equiv \sqrt{\psi_{m} /\left(27 \psi_{\theta}\right)}-1$, and satisfying $L\left(\bar{\Psi}_{m}\right)=U\left(\bar{\Psi}_{m}\right)$

This proposition is illustrated in Figure 1, which shows the set $\mathcal{M}$ in the $\left(\Psi_{m}, \Psi_{\theta}\right)$ plane. Multiple equilibria arise when $\left(\Psi_{\theta}, \Psi_{m}\right)$ lies in between the two boundaries. The boundaries are strictly positive. Thus, when $\left(\Psi_{m}, \Psi_{\theta}\right)$ is small enough, there is a unique equilibrium and a mild increase in the public information vector may create multiplicity.

Figure 2 shows graphically how changing public information distorts the fixed-point equation and can create multiple equilibria. For a low value of $\Psi_{m}$, complementarities are strong and the right-hand-side of the fixed-point equation, (31), has a sharply increasing S-shape; it has a unique intersection with the 45-degree line in the upper branch of the S. For a middle value of $\Psi_{m}$, the S-shape rises more slowly and three intersections arise. When $\Psi_{m}$ is large, the $\mathrm{S}$ becomes decreasing and a unique intersection is obtained. A similar graphical analysis applies to changes in $\Psi_{\theta}$.

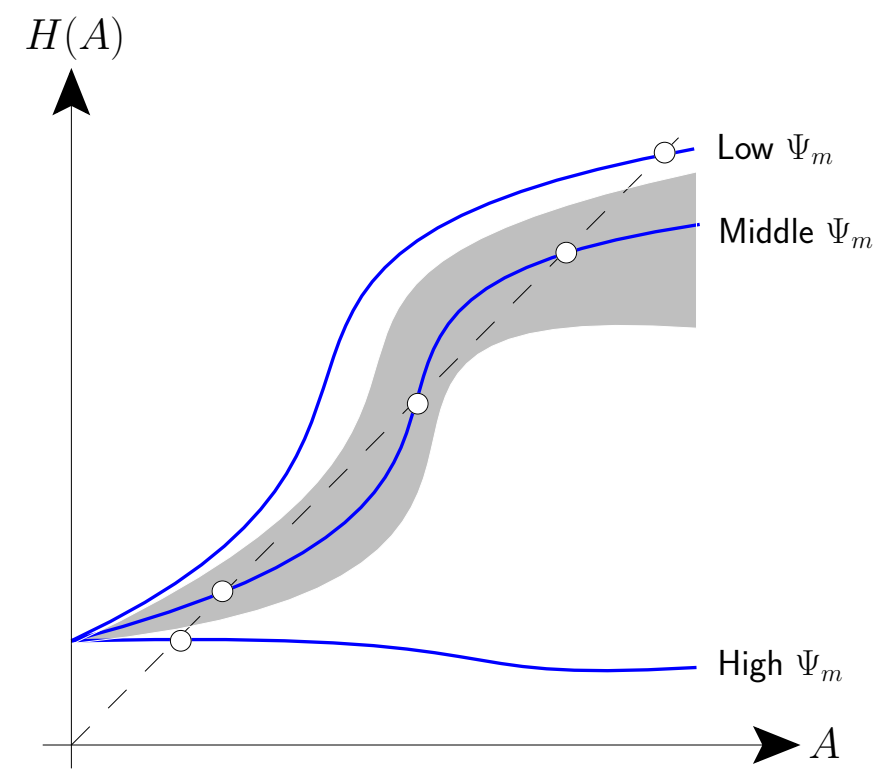

Figure 2: The impact of changing $\Psi_{m}$ on the fixed-point equilibrium equation. 
The following proposition accounts for all equilibria:

Proposition 4. Given $\left(\psi_{m}, \psi_{\theta}\right)$ such that $\psi_{m} / \psi_{\theta}>27$, then:

(i) for all $\left(\Psi_{m}, \Psi_{\theta}\right)$ in the interior of $\mathcal{M}$, there are three equilibria, $A_{L}<A_{M}<A_{H}$;

(ii) for all $\left(\Psi_{m}, \Psi_{\theta}\right)$ either on the upper or lower boundaries of $\mathcal{M}$, there are two distinct equilibria, $A_{L}<A_{H}$; for $\left(\bar{\Psi}_{m}, U\left(\bar{\Psi}_{m}\right)\right)$, there is only one;

(iii) as $\left(\Psi_{m}, \Psi_{\theta}\right)$ approaches the lower boundary from below, the unique equilibrium converges to $A_{H}$;

(iv) as $\left(\Psi_{m}, \Psi_{\theta}\right)$ approaches the upper boundary from above, the unique equilibrium converges to $A_{L}$.

Thus, in the interior of the multiple equilibrium region, $\mathcal{M}$, there are three equilibria. Note that there are two distinct equilibria on the boundaries of $\mathcal{M}$ but, just outside $\mathcal{M}$, there is a unique equilibrium. Hence, as one enters $\mathcal{M}$, at least one "new" equilibrium must appear. This is what is demonstrated by the third and fourth points of the proposition: a strictly lower equilibrium, $A_{L}$, appears as one enters $\mathcal{M}$ from below, and a strictly higher equilibrium, $A_{H}$, appears as one enters $\mathcal{M}$ from above.

\section{Public Information and Welfare}

This section analyzes the welfare impact of public information releases about $m$ and $\theta$. Public information has a direct beneficial effect: taking the aggregate weight, $A$, as given, it increases the total knowledge of the households and allows for more informed decisions. However, it also has the negative effect of reducing the weight that households put on their private information, which reduces the endogenous informational content of nominal prices. If $\psi_{m}>0$, so that prices generate private information, then the second effect can dominate.

\subsection{Welfare Criterion and Equilibrium Selection}

We take our criterion to be utilitarian welfare: the ex ante utility of a representative household. In our model, it turns out to be an increasing function of households' equilibrium posterior precision about $\theta$ : 
Proposition 5. In a linear equilibrium, ex-ante utilitarian welfare is an increasing function of the posterior precision, $\psi_{\theta}^{\prime}+\Psi_{\theta}^{\prime}=\psi_{\theta}+A^{2} \psi_{m}+\Psi_{\theta}+A^{2} \Psi_{m}$, about $\theta$.

Households' ex-ante welfare goes up with the total precision of their first-period beliefs, $\psi_{\theta}^{\prime}+\Psi_{\theta}^{\prime}$. This simply means that households are better off if they know more about $\theta$ when they make their labor supply decisions. Although intuitive, this result is not a forgone conclusion: as it is well known, information does not necessarily have a positive social value. ${ }^{9}$

Multiple equilibria introduce a standard difficulty for welfare analysis. Indeed, one has to decide which equilibrium households coordinate on, and different equilibria often admit opposite comparative statics. In this section we focus on the highest welfare equilibrium, so that we abstract from the negative welfare impact of coordination failure. Also, as will become clear later, our main welfare result does not depend on multiplicity: it also holds in regions of the parameter space where the equilibrium is unique. Proposition 5 immediately implies:

Lemma 3. The highest welfare equilibrium corresponds to the largest solution, $A_{\star}$, of the equilibrium fixed-point equation (31).

\subsection{A U-Shaped Welfare Function}

Recall first that, in equilibrium, the total posterior precision of a household is

$$
\psi_{\theta}^{\prime}+\Psi_{\theta}^{\prime}=\psi_{\theta}+A_{\star}^{2} \psi_{m}+\Psi_{\theta}+A_{\star}^{2} \Psi_{m}
$$

One sees that, holding $A_{\star}$ constant, both $\Psi_{\theta}$ and $\Psi_{m}$ increase public knowledge. This is the intuitive direct beneficial effect of public information: it directly increases knowledge about $\theta$, or it increases knowledge about money, $m$, which allows households to extract more information about $\theta$ from nominal prices.

There is, however, a countervailing equilibrium effect: following an increase in public information about either $\theta$ or $m$, households put less weight on their private knowledge, reducing the equilibrium weight $A_{\star}$. This indirect effect tends to decrease the informational content of prices:

\footnotetext{
${ }^{9}$ Perhaps the best known example is from Hirshleifer (1971), who shows that information destroys insurance opportunities. See, also, the first chapter of Brunermeier (2001) and the references therein.
} 
Lemma 4. In the highest welfare equilibrium, the equilibrium weight, $A_{\star}$ : (i) is strictly decreasing in $\Psi_{m}$ and $\Psi_{\theta}$, and goes to zero as either $\Psi_{m}$ or $\Psi_{\theta}$ go to infinity and (ii) is continuous in $\left(\Psi_{m}, \Psi_{\theta}\right)$ everywhere except at the upper boundary of $\mathcal{M}, \Psi_{\theta}=U\left(\Psi_{m}\right)$, where it jumps downwards (when moving from the interior of the set $\mathcal{M}$ to a point outside).

Note that, as $\Psi_{m}$ and $\Psi_{\theta}$ increase smoothly, when the negative jump in $A_{\star}$ occurs, society incurs a discrete welfare loss. Now, from the fixed-point equation, (31), one can write that,

$$
\psi_{\theta}+A_{\star}^{2} \psi_{m}+\Psi_{\theta}+A_{\star}^{2} \Psi_{m}=\frac{\psi_{\theta}}{A_{\star}}+A_{\star} \psi_{m},
$$

where the left-hand side is the total posterior precision of households' beliefs. A change in $\Psi_{m}$ or $\Psi_{\theta}$ affects the right hand side only through the effect on equilibrium $A_{\star}$. Hence, we can evaluate the welfare effect of an increase in $\Psi_{m}$ and/or $\Psi_{\theta}$ by taking derivatives of the right hand side with respect to $A_{\star}$ :

$$
\frac{\partial}{\partial A_{\star}}\left(\psi_{\theta}+A_{\star}^{2} \psi_{m}+\Psi_{\theta}+A_{\star}^{2} \Psi_{m}\right)=-\frac{\psi_{\theta}}{A_{\star}^{2}}+\psi_{m},
$$

which is positive if and only if $A_{\star}>\sqrt{\psi_{\theta} / \psi_{m}}$. Keeping in mind that $A_{\star}$ is strictly decreasing in $\left(\Psi_{m}, \Psi_{\theta}\right)$, we obtain:

Lemma 5. Suppose households coordinate on the highest welfare equilibrium and fix some $\left(\psi_{m}, \psi_{\theta}\right)$. Then, when $\Psi_{\theta} \neq U\left(\Psi_{m}\right)$, welfare decreases continuously in $\left(\Psi_{m}, \Psi_{\theta}\right)$ if and only if $A_{\star}>\sqrt{\psi_{\theta} / \psi_{m}}$. When $\Psi_{\theta}=U\left(\Psi_{m}\right)$, a marginal increase in $\left(\Psi_{m}, \Psi_{\theta}\right)$ causes welfare to jump down.

Equipped with this lemma, we can precisely characterize the region of the $\left(\Psi_{m}, \Psi_{\theta}\right)$ plane where welfare is decreasing in $\left(\Psi_{m}, \Psi_{\theta}\right)$. Let's define, for all $\Psi_{m} \geq 0$, the boundary

$$
\begin{aligned}
\Gamma\left(\Psi_{m}\right) & \equiv \sup \left\{\Psi_{\theta} \geq 0: A_{\star}>\sqrt{\frac{\psi_{\theta}}{\psi_{m}}}\right\} \\
& =0 \text { if the set is empty. }
\end{aligned}
$$

Then, because $A_{\star}$ is decreasing and left-continuous in $\left(\Psi_{m}, \Psi_{\theta}\right)$, it follows that $A_{\star} \geq \sqrt{\psi_{\theta} / \psi_{m}}$ if and only if $\Psi_{\theta} \leq \Gamma\left(\Psi_{m}\right)$. So welfare is decreasing when $\left(\Psi_{m}, \Psi_{\theta}\right)$ falls below the boundary $\Psi_{\theta}=\Gamma\left(\Psi_{m}\right)$. Precisely, by Lemma 5, when $\Psi_{\theta} \neq U\left(\Psi_{m}\right)$, welfare is continuously decreasing if and only if $\Psi_{\theta}<\Gamma\left(\Psi_{m}\right)$. When $\Psi_{\theta}=U\left(\Psi_{m}\right)$, welfare jumps down. 
In the appendix, we demonstrate that $\Gamma\left(\Psi_{m}\right)>0$ for some $\Psi_{m}$, so that the region below the boundary, $\Psi_{\theta}<\Gamma\left(\Psi_{m}\right)$, is in general not empty. In addition, we provide a parametric description of $\Gamma\left(\Psi_{m}\right)$ :

Proposition 6 (U-Shaped Welfare). Suppose that households coordinate on the highest welfare equilibrium and fix some $\left(\psi_{m}, \psi_{\theta}\right)$. Let $\Delta\left(\Psi_{m}\right)=2\left(\sqrt{\psi_{\theta} \psi_{m}}-\psi_{\theta}\right)-\left(\psi_{\theta} / \psi_{m}\right) \Psi_{m}$ and

$$
\begin{aligned}
\Gamma\left(\Psi_{m}\right) & =\max \left\{0, \Delta\left(\Psi_{m}\right), U\left(\Psi_{m}\right)\right\} & & \text { whenever } U\left(\Psi_{m}\right) \text { is defined } \\
& =\max \left\{0, \Delta\left(\Psi_{m}\right)\right\} & & \text { otherwise, }
\end{aligned}
$$

and where $\Delta\left(\bar{\Psi}_{m}\right) \geq U\left(\bar{\Psi}_{m}\right)$. Then, when $\Psi_{\theta} \neq U\left(\Psi_{m}\right)$, welfare decreases continuously in $\left(\Psi_{m}, \Psi_{\theta}\right)$ if and only if $\Psi_{\theta}<\Gamma\left(\Psi_{m}\right)$. When, $\Psi_{\theta}=U\left(\Psi_{m}\right)$, welfare jumps down.

The boundary $\Gamma\left(\Psi_{m}\right)$ is shown in Figure 3. Along any increasing curve in the $\left(\Psi_{m}, \Psi_{\theta}\right)$ plane that passes through the origin, welfare will have a $U$ shape: it will decrease first, reach a minimum when crossing the boundary $\Psi_{\theta}=\Gamma\left(\Psi_{m}\right)$ from below, and increase thereafter. Note also that the curve may cross the boundary $\Psi_{\theta}=U\left(\Psi_{m}\right)$ before or at the same time as the boundary $\Psi_{\theta}=\Gamma\left(\Psi_{m}\right)$. At that crossing point, the high equilibrium disappears and welfare will have a negative jump.

One may suspect that the negative welfare result only arises when there are multiple equilibria coupled with that fact that we have arbitrarily chosen to focus on the high welfare equilibrium. The proposition clarifies that it is not the case: welfare can decrease when increasing $\left(\Psi_{m}, \Psi_{\theta}\right)$ even in regions where the equilibrium is unique. For example, if $\psi_{\theta}<$ $\psi_{m}<27 \psi_{\theta}$, then the equilibrium is unique and the region of the $\left(\Psi_{m}, \Psi_{\theta}\right)$ plane where welfare decreases is non-empty.

Another implication of the proposition is that an optimal communication policy is bangbang:

Corollary 1 (Bang-Bang Communication). Suppose the government has multidimensional information about $m$ and $\theta$ that would permit an increase of public precisions. Then, the optimal communication policy is to announce all or none of the information.

This means that, in this setup, either full transparency or full opacity is optimal: selectively picking the information to announce, or revealing only part of the available information, will always be suboptimal. In Section 5.1, we qualify this results in an economy where 


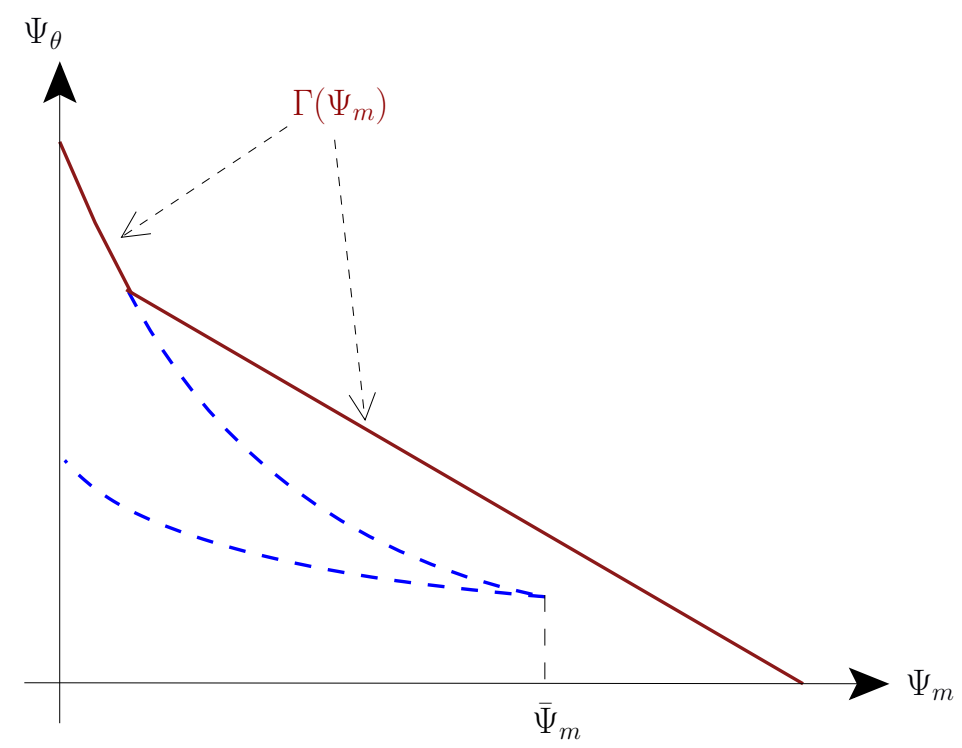

Figure 3: The solid line is the boundary $\Psi_{\theta}=\Gamma\left(\Psi_{m}\right)$ above which welfare decreases in $\left(\Psi_{m}, \Psi_{\theta}\right)$. The dotted line shows the boundaries of the set $\mathcal{M}$.

the shock $m$ is the sum of three components: a shock to a narrow monetary aggregate, a velocity shock, and an observational noise. We show that, for small releases of public information, the bang bang result only applies "component-by-component". For instance, it can be optimal to release public information about the narrow monetary aggregate, and not about the velocity shock.

The next proposition studies some conditions for transparency and opacity to be optimal:

Proposition 7. A sufficiently large release of public information about $\Psi_{m}$ or $\Psi_{\theta}$ will always increase welfare. On the other hand, for any given finite increase of public precisions, there exists $\left(\psi_{m}, \psi_{\theta}\right)$ such that this increase is welfare decreasing.

The first point of the proposition intuitively arises from the fact that, if $\Psi_{m}$ goes to infinity, then households are no longer confused about monetary shocks and they can extract a signal of arbitrarily high precision about $\Theta$ from the observation of prices; their posterior precision goes to infinity and welfare is maximized. Of course, if $\Psi_{\theta}$ goes to infinity, then the posterior precision goes to infinity as well (as information can never be destroyed). The second point of the Proposition follows from the parametric form of $\Gamma\left(\Psi_{m}\right)$ given in Proposition 6: in particular, if $\psi_{m}$ is large enough, then $\Psi_{\theta}<\Delta\left(\Psi_{m}\right) \leq \Gamma\left(\Psi_{m}\right)$, so that an increase of public information up to $\left(\Psi_{m}, \Psi_{\theta}\right)$ decreases welfare. 


\section{Explaining the Negative Welfare Result: The Role of Complementarities}

Recall that Lemma 5 showed that, in order for public information to reduce welfare, it is necessary and sufficient that $A_{\star}>\sqrt{\psi_{\theta} / \psi_{m}}$. Since $A_{\star} \leq 1$, it follows that a necessary condition for public information to reduce welfare is that $\psi_{m} \geq \psi_{\theta}$. In particular, we must have that $\psi_{m}>0$, meaning that the distribution of price must generate private information. In Amador and Weill (2006), we obtained a similar result in an abstract model of information diffusion: private learning from other agents' actions is necessary for public information to be welfare reducing.

In order to clarify this point and highlight the importance of the endogenous private information generated by prices, we first show that, in the absence of an effect on endogenous private information, public information is always beneficial:

Lemma 6. Holding $\psi_{\theta}^{\prime} \equiv \psi_{\theta}+A_{\star}^{2} \psi_{m}$ the same,

(i) the equilibrium weight $A_{\diamond}$ which solves

$$
A_{\diamond}=H\left(\psi_{\theta}^{\prime}, \Psi_{\theta}+A_{\diamond}^{2} \Psi_{m}\right)
$$

is a decreasing function of $\Psi_{m}$ and $\Psi_{\theta}: \partial A_{\diamond} / \partial \Psi_{m}<0$ and $\partial A_{\diamond} / \partial \Psi_{\theta}<0$, and

(ii) the posterior precision $\psi_{\theta}^{\prime}+\Psi_{\theta}+A_{\diamond}^{2} \Psi_{m}$ is an increasing function of $\Psi_{m}$ and $\Psi_{\theta}$.

The lemma shows that, when $\psi_{\theta}^{\prime}$ is held constant, although public information about $m$ or $\theta$ reduces the equilibrium sensitivity $A_{\diamond}$, it always increases the posterior precision $\psi_{\theta}^{\prime}+\Psi_{\theta}+A_{\diamond}^{2} \Psi_{m}$. The intuition for this result is as follows. The posterior public precision, $\Psi_{\theta}+A_{\diamond}^{2} \Psi_{m}$, cannot decrease with an increase in $\left(\Psi_{m}, \Psi_{\theta}\right)$ given that $\psi_{\theta}^{\prime}$ remained constant. Indeed, if the posterior public precision had decreased, households would find it optimal to rely more on their private information, so the equilibrium $A_{\diamond}$ would have to increase. This implies, in turn, an increase in $\Psi_{\theta}+A_{\diamond}^{2} \Psi_{m}$, contradicting the assumed decrease in public knowledge.

It follows also from the above lemma that, for public information to be welfare reducing, a higher reduction in the equilibrium $A_{\star}$ needs to be generated. This is where the complementarities provide a powerful amplification mechanism. Consider, for instance, an increase in $\Psi_{m}$. This causes $A$ to decrease, which always decreases the amount $A^{2} \psi_{m}$ of private 
information generated by price. This prompts households to rely less on their private information, i.e., to lower $A$, which decreases $A^{2} \psi_{m}$ further, prompts households to lower $A$, and so on.

Informally, by applying the implicit function theorem (while assuming differentiability) to the equilibrium fixed-point equation (31), we find that the marginal change in the equilibrium weight when $\Psi_{m}$ increases is given by

$$
\frac{\partial A_{\star}}{\partial \Psi_{m}}=\frac{A_{\star}^{2} H_{\Psi_{\theta}^{\prime}}}{1-2 A_{\star}\left(H_{\Psi_{\theta}^{\prime}} \Psi_{m}+H_{\psi_{\theta}^{\prime}} \psi_{m}\right)}<\frac{A_{\star}^{2} H_{\Psi_{\theta}^{\prime}}}{1-2 A_{\star} H_{\Psi_{\theta}^{\prime}} \Psi_{m}}=\frac{\partial A_{\diamond}}{\partial \Psi_{m}}<0,
$$

where $H_{\psi_{\theta}^{\prime}}>0$ and $H_{\Psi_{\theta}^{\prime}}<0$ are the partial derivatives of $H$ with respect to $\psi_{\theta}^{\prime}$ and $\Psi_{\theta}^{\prime}$, respectively. Because of the complementarities in actions created by the endogenous private information, the equilibrium sensitivity $A_{\star}$ falls by more than if $\psi_{\theta}^{\prime}$ had remained constant. This can reduce the total amount $A^{2} \psi_{m}+A^{2} \Psi_{m}$ of information generated by prices, even if $\Psi_{m}$ increases.

\subsection{Related Results from the Literature}

Perhaps the best known related result is that of Morris and Shin (2002), who have shown, in the context of a beauty contest game, that public information can reduce welfare. Although reminiscent of their result, ours does not arise from agents' socially wasteful desire to coordinate their actions, but instead from the endogenous aggregation of information through prices. In particular, in Morris and Shin (2002), public information always reduces agents' uncertainty about fundamentals, while in our model it can have the opposite effect (and this is the driver of our welfare result).

Morris and Shin (2002) emphasize that releases of public information are welfare decreasing when the precision of the private information about the aggregate state of the economy is sufficiently large. In our economy, where the aggregate state has two dimensions, $m$ and $\theta$, this result does not always hold. For instance, consider the case when $\Psi_{\theta}=0$. Then, the equilibrium is unique and an increase in $\Psi_{m}$ reduces welfare if and only if

$$
\Psi_{m} \leq 2 \psi_{m}\left(\sqrt{\frac{\psi_{m}}{\psi_{\theta}}}-1\right)
$$


One sees that, as in Morris and Shin (2002), a larger $\psi_{m}$ increases the range where public announcements are welfare decreasing. A larger $\psi_{\theta}$, however, has the opposite effect.

In Svensson's (2006) critique of Morris and Shin (2002), it is proposed that a conservative benchmark of how likely it is that public information is welfare reducing is when the precision of the public and the private signals are the same. Indeed, in practice, one would expect the monetary authority to know at least as much about economic shocks as the private sector. When imposing this restriction in Morris and Shin (2002)'s model, Svensson (2006) finds that public information is welfare increasing. He concludes that Morris and Shin (2002) are, in fact, pro-transparency. In our multidimensional economy, let us interpret Svensson's restriction as letting $\Psi_{m}=\psi_{m}$ and $\Psi_{\theta}=\psi_{\theta}$. From Proposition 6 , in order for a public release of information to reduce welfare, it is sufficient that $\Psi_{\theta} \leq \Delta\left(\Psi_{m}\right)$ or, after rearranging:

$$
\frac{\Psi_{m}}{2 \psi_{m}}+\frac{\Psi_{\theta}}{2 \psi_{\theta}}<\sqrt{\frac{\psi_{m}}{\psi_{\theta}}}-1
$$

which, when $\Psi_{m}=\psi_{m}$ and $\psi_{\theta}=\Psi_{\theta}$, is equivalent to $\psi_{m}>4 \psi_{\theta}$. If this condition is satisfied, then, public information about $m$ decreases welfare even though the precision of the public and private signals about $m$ are the same.

Some recent papers have shown the reappearance of multiple equilibria in global games if information is publicly aggregated through prices (see Angeletos and Werning (2006) and Hellwig, Mukherji and Tsyvinski (2006)). It is worth noticing that, differently from these papers (and global games in general), in our current model multiplicity is generated even though there are no payoff externalities and, under full information, our model will always feature a unique equilibrium. Also, the focus of our analysis is not to study the multiplicity in itself, but instead to understand the role of public information releases on welfare and uncertainty.

Lastly, our paper is related to the recent work of Ganguli and Yang (2007) who study information acquisition in a noisy rational expectations financial market model. Under the assumption that investors have private information about the amount of noise trading, they also find that their model may admit multiple equilibria. This is formally similar to our assumption that households have private information about the money supply. Note however, that our main results do not concern the multiplicity itself, but welfare analysis (which include situations where the set of equilibria is a singleton) and how multiplicity is 
affected by public announcements.

\section{Extensions}

In this last section, we provide three extensions of our model. In Subsection 5.1, we add a velocity shock to the model in order to demonstrate that $M$ can indeed be interpreted as a broad monetary aggregate. Also, we show how our model can easily accommodate multiple shocks. In Subsection 5.2, we open a centralized financial market for a one-period nominal bond and show that all our results go through. In Subsection 5.3 we introduce an large idiosyncratic component to the agents' decisions, and show that our results are not affected.

\subsection{Narrow versus Broad Monetary Aggregates}

The objective of the exposition so far has been to clarify the forces at play and to gain an understanding of the mechanism through which public information can be welfare reducing. For that reason, we have been imprecise about what the monetary shock $m$ stands for. In this section, we provide an extension of our model where the shock $m$ is the sum of three underlying shocks: a shock to a narrow monetary aggregate, a velocity shock that represents uncertainty about credit conditions, and an observational noise to capture the noise in preliminary price level statistics. We then proceed to derive a general condition for the marginal release of information regarding either one of these three shocks to be welfare increasing: we find that the release of marginal public information about a shock is welfare increasing if the ratio of public to private knowledge about that particular shock is sufficiently small. Thus, for small release of public information, our bang-bang communication result applies component by component: for instance, it can be optimal to release information about the narrow monetary aggregate, but not about the velocity shock.

\section{The Model}

We begin by distinguishing between narrow and broad monetary aggregates through the introduction of velocity shocks: we let the price level in every location not only depend on the local money endowment $M_{i}$, but also on some level of velocity, $V_{i}$. Precisely, in the equilibrium to be described, the amount of liquidity that enters the quantity equation is not $M_{i}$, but the product $M_{i} V_{i}$, which we interpret as a broad monetary measure. We 
assume that the monetary authority has complete information about the distribution of money endowments, $M_{i}$. This represents disaggregated information that the Central Bank collects about narrow measures of money, such as the monetary base. On the other hand, we assume that the Central Bank has imperfect information about the velocity shocks, $V_{i}$. These could represent, for instance, partial information about overall credit conditions in the economy.

The representative household in location $i$ faces a CIA constraint of the form:

$$
C_{i t} \leq V_{i}\left(\frac{M_{i t-1}^{d}+T_{i t}}{P_{i t}}\right)
$$

where $T_{i t}$ are nominal transfers made by the government to household $i$ at the beginning of period $t$, and $V_{i}$ represent a local velocity parameter. A standard interpretation of this velocity parameter is that a household can use credit to purchase a fraction $\left(1-1 / V_{i}\right)$ of its consumption goods, but has to purchase the rest of the goods with cash. According to this interpretation, $V_{i}$ is positively related to the amount of inside money created in location $i$. The budget constraint of a household is

$$
C_{i t}+\frac{M_{i t}^{d}}{P_{i t}} \leq L_{i t}+\frac{M_{i t-1}^{d}+T_{i t}}{P_{i t}}
$$

For tractability, we assume that the Central Bank implements the Friedman rule ${ }^{10}$ by shrinking the money supply at rate $\beta$ : we assume that at time $t$, households in location $i$ have to pay a lump sum tax $T_{i t}=-(1-\beta) \beta^{t-1} M_{i}$. We let the Central Bank know the distribution of the initial cash holdings so it has sufficient information to implement this policy. Note as well that the Friedman rule is optimal in this economy from period 2 onwards. ${ }^{11}$

Finally, the goods market clearing conditions are, as before, (7), but the money market clearing condition becomes

$$
M_{i t}^{d}=\beta^{t} M_{i}
$$

\footnotetext{
${ }^{10}$ It is possible to solve for an equilibrium under the assumption that the money supply is constant. We would need to modify the distributional assumptions on the velocity shocks to guarantee that the model remains log-normal. These changes would not affect the bottom-line of the results that follow. The details of this exercise are available from the authors upon request.

${ }^{11}$ Whether the Friedman rule is optimal in period 1 remains to be shown. The difficulty relates to footnote 10: changes in the monetary rule require changes in distributional assumptions for the model to remain log-normal.
} 
so that $\int M_{i t}^{d} d i=\beta^{t} M=\beta^{t} \int M_{i} d i$, the initial aggregate money stock, which accounts for the ever shrinking aggregate money supply.

Each of the households is initially endowed with the same information about $\Theta$ and $M$ as before.

The velocity parameter in each location is given by $\log V_{i} \equiv \hat{v}_{i}=v+\varepsilon_{v i}$, where $\varepsilon_{v i}$ is normally distributed with a mean of zero and a precision $\psi_{v}$, and where $v$ captures an aggregate velocity shock. In addition, households share the common prior that the average velocity, $v$, is normally distributed ${ }^{12}$ with a mean of zero and a precision $\Psi_{v}$.

Households do not observe the aggregate values $m$ or $v$, which are assumed to be uncorrelated. However, they imperfectly observe the average price level in the economy; that is, they observe $p+\varepsilon_{p}$, where $p$ is the log average price and $\varepsilon_{p}$ is a normal noise with mean zero and precision $\Psi_{p}$, and independent of everything else.

As before, public releases of information boil down to increases in the public precisions $\Psi_{m}, \Psi_{v}$, and $\Psi_{p}$. If the Central Bank releases its perfect information about $m$, then it can increase the corresponding public precision, $\Psi_{m}$, towards infinity. The monetary authority, however, does not have full information regarding the velocity shock: it can only increase $\Psi_{v}$ up to some finite amount.

Optimization by the households, together with the market-clearing conditions, implies that in equilibrium

$$
\begin{aligned}
L_{i t} & =C_{i t}=\mathbb{E}_{i t}[\Theta]^{-1} \\
P_{i t} & =\beta^{t} \mathbb{E}_{i t}[\Theta] V_{i} M_{i} \\
M_{i t}^{d} & =\beta^{t} M_{i}
\end{aligned}
$$

Once $\Theta$ is revealed in period 2, the equilibrium is deterministic, so the only interesting behavior occurs in period 1 (as in the baseline model). The definition of equilibrium is the same as in Definition 1, except that we now require that the average log price be

$$
p=a_{0}+a_{1} \theta+a_{2} m+a_{3} v+a_{4} \varepsilon_{p}
$$

that the conditional expectations include $v_{i}$ as part of the information sets and that equa-

\footnotetext{
${ }^{12}$ Since $V_{i}$ is log-normally distributed in the cross section, $V_{i}$ can be smaller than 1 , implying that the fraction of credit goods, $1-1 / V_{i}$, may be less than zero. However, by choosing the mean of $V_{i}$ large enough, we can make this happen with arbitrarily small probability.
} 
tions (16) and (17) are replaced by equations (36) and (37). In the appendix, we guess that observing the price with noise is observationally equivalent to $\hat{p}=\theta+m / A+v / B+\varepsilon_{p} / C$, and show that:

Proposition 8. There exists a linear equilibrium. In a linear equilibrium, the total precision of the posterior about $\theta$ solves the following equation:

$$
\kappa=G(\kappa) \equiv \psi_{\theta}+\Psi_{\theta}+\left[\frac{1}{A^{2}\left(\psi_{m}+\Psi_{m}\right)}+\frac{1}{B^{2}\left(\psi_{v}+\Psi_{v}\right)}+\frac{1}{C^{2} \Psi_{p}}\right]^{-1},
$$

where

$$
A=\frac{\psi_{\theta}}{\kappa}+\frac{1-\left(\psi_{\theta}+\Psi_{\theta}\right) / \kappa}{1+\Psi_{m} / \psi_{m}} ; B=\frac{\psi_{\theta}}{\kappa}+\frac{1-\left(\psi_{\theta}+\Psi_{\theta}\right) / \kappa}{1+\Psi_{v} / \psi_{v}} ; C=\frac{\psi_{\theta}}{\kappa} .
$$

Conversely, every solution where $\kappa \in\left(\psi_{\theta}+\Psi_{\theta}, \infty\right)$ corresponds to a linear equilibrium.

It can be shown that the linear equilibria in this model converge to the ones in our baseline model as $\Psi_{v}$ and $\Psi_{p}$ tend towards infinity. Similarly as in the baseline model, there is the possibility of multiple equilibria. We focus, as before, then on characterizing the equilibrium corresponding to the highest fixed point $\kappa_{\star}$ of $G(\kappa)$, which delivers the highest welfare.

\section{Optimal Communication}

By analyzing how the function $G(\kappa)$ varies with the public precisions $\Psi_{m}, \Psi_{v}$, and $\Psi_{p}$, we can show that,

Proposition 9. A marginal release of information about shock $i \in\left\{m, v, \varepsilon_{p}\right\}$ is welfare reducing at the best equilibrium, $\kappa_{\star}$ iff

$$
\frac{\Psi_{i}}{\psi_{i}}<\frac{\kappa_{\star}-\Psi_{\theta}}{\psi_{\theta}}-2
$$

The release of marginal public information about a shock is welfare increasing if the ratio of public to private knowledge about that particular shock is sufficiently small. A direct corollary is that it is always beneficial to release public information about shocks that the households have no private information about. This implies that improving the observation of the price, by increasing the precision of the noise $\varepsilon_{p}$, always increases welfare.

Another result that follows is that if a benevolent public agency has full information about any shock, it should announce it: 
Proposition 10. Revealing the realization of any shock $i \in\left\{m, v, \varepsilon_{p}\right\}$ increases the precision of households' beliefs about $\theta$ and increases welfare.

Hence the Central Bank should announce the state of the narrow monetary aggregate. Also the Central Bank should try to ensure that the aggregate price level is observed as efficiently as possible by the agents. Given this, whether the publication of a signal about the nominal output (or equivalently, about the velocity shock) is welfare improving or not depends on the conditions stated in Proposition 9. In particular, if $\Psi_{p}=\infty$, so the price level is observed without noise, the model thus converges to our baseline model, with only two shocks: $\theta$ and $v$.

\subsection{A Bond Market: No Trade and No Information}

A familiar way in which an economy aggregates dispersed private information is through financial markets. One might wonder, then, how robust the results regarding the social value of public announcements that we have obtained are to the introduction of a financial market where households from different locations can interact. To answer this question, we introduce what we believe is a natural financial market in our economy: households are allowed to trade a nominal bond in zero net supply. Our main result is that the equilibrium nominal interest rate in that market does not provide any new information to the households and that the allocation obtained by a competitive equilibrium when the bond market is closed remains the allocation of a competitive equilibrium once it is opened.

Thus, suppose that any household at period $t$ can buy a bond that pays a unit of the currency in the following period, $t+1$, and let us denote by $Q_{t}$ its nominal price. The budget constraint of the household $i$ in period $t$ is now given by

$$
C_{i t}+\frac{M_{i t}^{d}}{P_{i t}}+\frac{B_{i t}}{P_{i t}} Q_{t} \leq L_{i t}+\frac{M_{i t-1}^{d}}{P_{i t}}+\frac{B_{i t-1}}{P_{i t}}
$$

where $B_{i t}$ is the amount of the bond held by household $i$ in period $t$. As before, the household is subject to a cash-in-advance constraint.

The bond market clearing condition is, at all times:

$$
\int B_{i t} d i=0 .
$$


In an equilibrium, from the Euler equation of household $i$, we obtain that

$$
Q_{t}=\beta \mathbb{E}_{i t}\left[\frac{u^{\prime}\left(C_{i t+1}\right)}{u^{\prime}\left(C_{i t}\right)} \frac{P_{i t}}{P_{i t+1}}\right]
$$

We now check that the allocation without a nominal bond market remains an equilibrium once the nominal bond market opens. Because the CIA constraint binds at all times, $P_{i t} C_{i t}=$ $M_{i}$, and hence,

$$
Q_{t}=\beta \mathbb{E}_{i t}\left[\frac{C_{i t} P_{i t}}{C_{i t+1} P_{i t+1}}\right]=\beta,
$$

which is the same for all agents. Note as well that the price of the bond does not reveal any information: it is just equal to the discount factor. Thus, any equilibrium allocation when the bond market is closed remains an equilibrium when the bond market is open with $B_{i t}=0$ and $Q_{t}=\beta$.

Our result is obtained because our economy satisfies the conditions for a no-trade equilibrium that Constantinides and Duffie (1996) famously pointed out in their incomplete market model. First, households have homothetic utility and second, in equilibrium, the logarithm of local consumptions and local prices

$$
\begin{aligned}
\log C_{i t} & =-\mathbb{E}_{i t}[\theta]+\log \beta-\frac{1}{2 \operatorname{var}_{t}[\theta]} \\
\log P_{i t} & =\hat{m}_{i}-\log C_{i t}
\end{aligned}
$$

follow identically distributed random walks with drifts. Indeed, the first term is a martingale, because it is the conditional expectation of a fixed random variable, $\theta$. The drift, given by the second and the third term, is the same across locations because all households share the same posterior precision. Taken together, these imply that the expectation of the nominal stochastic discount factor, $\left(P_{i t+1} / P_{i t}\right)\left(C_{i t+1} / C_{i t}\right)$, is constant across locations.

\subsection{Large idiosyncratic variations in price}

In the model we have described so far, idiosyncratic variation in prices is generated from the private information regarding the aggregate disturbances. A recent literature has documented that the variation in the idiosyncratic component of prices (and other quantities) is an order of magnitude bigger than the variation of the aggregate components. Hence, one may argue that the main concern of economic agents is not forecasting macroeconomic 
shocks, but instead responding to idiosyncratic shocks. In this section, we address this concern explicitly by introducing idiosyncratic shocks into the model, and showing that our welfare results are consistent with arbitrarily large levels of idiosyncratic volatility.

Suppose that the productivity parameter now includes a perfectly observed idiosyncratic component. That is, let the utility of household in location $i$ be given by

$$
\mathbb{E}_{i 1}\left[\sum_{t=1}^{\infty} \beta^{t-1}\left(\log \left(C_{i t}\right)-\hat{\Theta}_{i} L_{i t}\right)\right]
$$

where now $\hat{\Theta}=\Theta e^{g_{i}}$ is a combination of an aggregate component $\Theta$, which is as before, and an idiosyncratic component $g_{i}$, generated from a normal distribution with mean zero and constant variance, and independent across locations. We assume that the individual realization of $g_{i}$ is observed perfectly by households in location $i$.

Following the same steps as in the previous sections, we obtain an optimality condition:

$$
C_{i t}=L_{i t}=\beta\left(\mathbb{E}_{i t} \hat{\Theta}_{i}\right)^{-1}=\beta e^{-g_{i}}\left(\mathbb{E}_{i t} \Theta\right)^{-1}
$$

Substituting this into the ex-ante (per period) welfare function:

$$
\mathbb{E}_{0}\left(\log \left(C_{i t}\right)-\hat{\Theta}_{i} L_{i t}\right)=\mathbb{E}_{0} \log \mathbb{E}_{i t}[\Theta]+\mathbb{E}_{0}\left[g_{i}\right]+\log \beta-\beta=\mathbb{E}_{0} \log \mathbb{E}_{i t}[\Theta]+\log \beta-\beta
$$

which delivers the same welfare function as the one obtained in section 4 . The equilibrium prices are then given by:

$$
\log P_{i t}=-\log \beta+\hat{m}_{i}+\log \mathbb{E}_{i t}[\Theta]+g_{i}=-\log \beta+\hat{m}_{i}+g_{i}+\mathbb{E}_{i}[\theta]+\frac{1}{2\left(\psi_{\theta}^{\prime}+\Psi_{\theta}^{\prime}\right)}
$$

Given that $g_{i}$ is idiosyncratic, its effect disappears in the aggregate, and the average log price in the economy is the same as the obtained in the baseline model. This implies that the equilibrium has not changed; and the results of Propositions 6 and 7 hold unmodified.

Note that according to the price equation we have obtained above, any level of idiosyncratic variation can now be added to the model by changing the variance of $g_{i}$ without affecting the welfare results. We interpret this as following: the fact that there is a large idiosyncratic component to the individual decisions do not imply that the welfare effects of announcements regarding the underlying aggregate shocks are necessarily trivialized. In particular, in the present example, idiosyncratic volatility does not affect at all the welfare 
calculations.

\section{Conclusions}

We have characterized the conditions under which public announcements about real and nominal aggregate shocks reduce the informativeness of prices and may actually increase uncertainty about fundamentals and lower welfare. Although we focus on the case where households observe nominal prices, we think it is reasonable to conjecture that a similar outcome will prevail in the presence of financial markets that also aggregate dispersed information in the economy.

Our model is basically static (the infinite horizon was just necessary for money to have value). However, similar techniques as the ones developed here may prove useful in studying the dynamic effects of information releases, and also in answering the timing question: when to make public announcements. This is all left for future research. 


\section{A Proofs}

\section{A.1 Proof of Proposition 1}

The right hand side of the fixed point equation (31) is strictly positive when $A=0$ and strictly less than 1 when $A=1$. Hence, it follows that equation (31) has at least one solution, $A_{\star}$. Plugging $\mathbb{E}[\theta \mid \hat{z}]=\hat{z}=A_{\star}^{2} \Psi_{m} /\left(\Psi_{\theta}+A_{\star}^{2} \Psi_{m}\right)\left(\theta+m / A_{\star}\right)$ back into equation (29), we see that our linear guess that $p=\alpha_{0}+\alpha_{1} \theta+\alpha_{2} m$ is verified for $\alpha_{0}=-\log \beta+1 / 2\left(\psi_{\theta}^{\prime}+\Psi_{\theta}^{\prime}\right)$, $\alpha_{1}=\left(A_{\star} \Psi_{\theta}+A_{\star}^{2} \Psi_{m}\right) /\left(\Psi_{\theta}+A_{\star}^{2} \Psi_{m}\right)$, and $\alpha_{2}=\alpha_{1} / A_{\star}$. Also, $A=A_{\star}$ uniquely determines the cross-sectional distribution of log prices, which is normal with a constant dispersion, as can be seen from equation (28) after substituting in for equations (26), $\psi_{\theta}^{\prime}=\psi_{\theta}+A_{\star}^{2} \psi_{m}$ and $\Psi_{\theta}^{\prime}=\Psi_{\theta}+A_{\star}^{2} \Psi_{m}$. Also, $A_{\star}$ determines the cross-sectional distribution of mean beliefs as implied by (26). Finally this determines a unique distribution for consumption and labor supplies, according to (16). This proves that $A_{\star}$ determines a unique equilibrium.

\section{A.2 Proof of Propositions 2, 3, and 4}

We start from the equilibrium equation (31):

$$
\begin{aligned}
& A=\frac{\psi_{\theta}+A^{2} \psi_{m}}{\psi_{\theta}+A^{2} \psi_{m}+\Psi_{\theta}+A^{2} \Psi_{m}} \\
\Leftrightarrow & A^{3}\left(\psi_{m}+\Psi_{m}\right)-A^{2} \psi_{m}+A\left(\psi_{\theta}+\Psi_{\theta}\right)-\psi_{\theta}=0 \\
\Leftrightarrow & A^{2}\left(\psi_{m}+\Psi_{m}\right)-A \psi_{m}+\left(\psi_{\theta}+\Psi_{\theta}\right)-\frac{\psi_{\theta}}{A}=0 \\
\Leftrightarrow & \frac{\Psi_{\theta}}{\psi_{\theta}}=-A^{2} \frac{\psi_{m}}{\psi_{\theta}}\left(1+\frac{\Psi_{m}}{\psi_{m}}\right)+A \frac{\psi_{m}}{\psi_{\theta}}-1+\frac{1}{A} \\
\Leftrightarrow & \rho_{\theta}=\lambda\left(-A^{2}\left(1+\rho_{m}\right)+A\right)+\frac{1}{A}-1 \\
\Leftrightarrow & \rho_{\theta} \equiv G\left(A, \rho_{m}\right),
\end{aligned}
$$

where $\lambda \equiv \psi_{m} / \psi_{\theta}, \rho_{\theta} \equiv \Psi_{\theta} / \psi_{\theta}, \rho_{m} \equiv \Psi_{m} / \psi_{m}$, and $G\left(A, \rho_{m}\right)$ depends implicitly on $\lambda$. Straightforward calculations show that

(R1) $G\left(A, \rho_{m}\right)$ goes to infinity when $A$ goes to zero;

(R2) $G\left(A, \rho_{m}\right)$ is negative when $A<0$ and $A \geq 1$. 
After taking derivatives, we find that $\partial G / \partial A=D\left(A, \rho_{m}\right) / A^{2}$, where

$$
\begin{aligned}
D\left(A, \rho_{m}\right) & =-2 \lambda\left(1+\rho_{m}\right) A^{3}+\lambda A^{2}-1 \\
\frac{\partial D}{\partial A} & =2 \lambda A\left[1-3 A\left(1+\rho_{m}\right)\right] .
\end{aligned}
$$

It thus follows that $D\left(A, \rho_{m}\right)$ is a hump-shaped function of $A$, is negative when $A=0$ and $A=1$, and achieves a maximum at $A=1 /\left[3\left(1+\rho_{m}\right)\right]$ :

$$
\max _{A \in[0,1]} D\left(A, \rho_{m}\right)=D\left(\frac{1}{3\left(1+\rho_{m}\right)}, \rho_{m}\right)=-1+\frac{\lambda}{27\left(1+\rho_{m}\right)^{2}} .
$$

It then follows that the function $G\left(A, \rho_{m}\right)$ is strictly decreasing in $A \in(0,1)$ if and only if the maximum of $D\left(A, \rho_{m}\right)$ is negative. Precisely:

(R3) If $\lambda \leq 27$ or $\left(\lambda>27\right.$ and $\left.\rho_{m} \geq \sqrt{\lambda / 27}-1\right)$, then $G\left(A, \rho_{m}\right)$ is strictly decreasing in $A \in(0,1)$.

(R4) If $\lambda>27$ and $\rho_{m}<\sqrt{\lambda / 27}-1$, then there exists

$$
0<a_{1}\left(\rho_{m}\right)<\frac{1}{3\left(1+\rho_{m}\right)}<a_{2}\left(\rho_{m}\right)<1
$$

such that $G\left(A, \rho_{m}\right)$ is decreasing in $A \in\left(0, a_{1}\left(\rho_{m}\right)\right)$, increasing in $A \in\left(a_{1}\left(\rho_{m}\right), a_{2}\left(\rho_{m}\right)\right)$, and decreasing again in $a \in\left(a_{2}\left(\rho_{m}\right), 1\right)$.

We also have:

(R5) The function $a_{1}\left(\rho_{m}\right)$ is increasing in $\rho_{m}$ and the function $a_{2}\left(\rho_{m}\right)$ is decreasing in $\rho_{m}$.

(R6) As $\rho_{m}$ approaches $\bar{\rho}_{m} \equiv \sqrt{\lambda / 27}-1$, both $a_{1}\left(\rho_{m}\right)$ and $a_{2}\left(\rho_{m}\right)$ tend to $1 /\left[3\left(1+\bar{\rho}_{m}\right)\right]$.

Result (R5) follows from an application of the Implicit Function Theorem (IFT). To prove (R6), note first that, by (R5), since $a_{i}\left(\rho_{m}\right)$ is monotonic and bounded, it must have a limit $\bar{a}_{i}$ as $\rho_{m}$ approaches $\bar{\rho}_{m}$. By continuity of $D\left(A, \rho_{m}\right)$, we must have that $D\left(\bar{a}_{i}, \bar{\rho}_{m}\right)=0$. Now, note that when $\rho_{m}=\bar{\rho}_{m}$, this equation has the unique solution $A=1 /\left[3\left(1+\bar{\rho}_{m}\right)\right]$. Thus, we must have that $\bar{a}_{i}=1 /\left[3\left(1+\bar{\rho}_{m}\right]\right.$, which proves the claim.

Now let us combine results (R2)-(R6) to find all solutions of the equation $\left.G\left(A, \rho_{m}\right)=\rho_{\theta}\right)$. First note that (R1) and (R2) show that there exists at least one solution, and that all solutions lie in the open interval $(0,1)$. In case (R3), the function $G\left(A, \rho_{m}\right)$ is strictly 
decreasing in $A$, so the solution is unique. To analyze the second case, (R4), we start by defining $B^{(1)}\left(\rho_{m}\right) \equiv G\left(a_{1}\left(\rho_{m}\right), \rho_{m}\right)$ and $B^{(2)}\left(\rho_{m}\right) \equiv G\left(a_{2}\left(\rho_{m}\right), \rho_{m}\right)$. We have

(R7) The function $B^{(i)}\left(\rho_{m}\right)$ is strictly decreasing in $\rho_{m}$, for $i \in\{1,2\}$.

Indeed, since by construction $\partial G / \partial A\left(a_{i}, \rho_{m}\right)=0$, it follows that, for $i \in\{1,2\}$ :

$$
\frac{d B^{(i)}}{d \rho_{m}}=\frac{\partial G}{\partial \rho_{m}}\left(a_{i}\right)=-\lambda a_{i}^{2}<0 .
$$

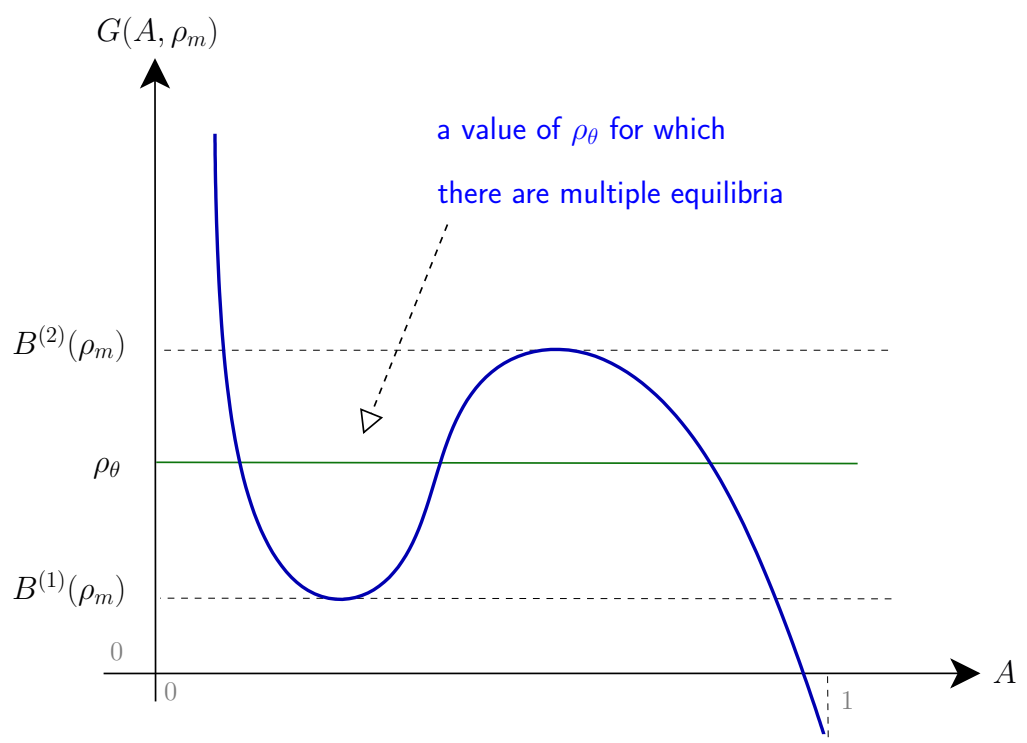

Figure 4: The function $G(A)$.

Now the set of equilibria can be described graphically, as follows. Figure 4 shows a plot of $G\left(A, \rho_{m}\right)$ as a function of $A$. The local minimum is achieved at $A=a_{1}$ and is equal to $B^{(1)}\left(\rho_{m}\right)$, and the local maximum is achieved at $a_{2}$ and is equal to $B^{(2)}\left(\rho_{m}\right)$. The figure makes it clear that

(R8) if $\rho_{\theta}<B^{(1)}\left(\rho_{m}\right)$, then there is a unique equilibrium, which is greater than $a_{2}\left(\rho_{m}\right)$;

(R9) if $\rho_{\theta}=B^{(1)}\left(\rho_{m}\right)$, then there are two equilibria, $A_{L}=a_{1}\left(\rho_{m}\right)<a_{2}\left(\rho_{m}\right)<A_{H}$;

(R10) if $B^{(1)}\left(\rho_{m}\right)<\rho_{\theta}<B^{(2)}\left(\rho_{m}\right)$, then there are three equilibria $A_{L}<a_{1}\left(\rho_{m}\right)<A_{M}<$ $a_{2}\left(\rho_{m}\right)<A_{H}$

(R11) if $\rho_{\theta}=B^{(2)}\left(\rho_{m}\right)$, then there are two equilibria, $A_{L}<a_{1}\left(\rho_{m}\right)<a_{2}\left(\rho_{m}\right)=A_{H}$; 
(R12) if $\rho_{\theta}>B^{(2)}\left(\rho_{m}\right)$, then there is a unique equilibrium, which is smaller than $a_{1}$.

Replacing $\rho_{m}=\Psi_{m} / \psi_{m}, \rho_{\theta}=\Psi_{\theta} / \psi_{\theta}$ and $\lambda=\psi_{m} / \psi_{\theta}$ in the above, we find that the set $\mathcal{M}$ is described parametrically by:

$$
\begin{aligned}
& 0 \leq \Psi_{m}<\bar{\Psi}_{m} \\
& \text { and } \quad L\left(\Psi_{m}\right) \leq \Psi_{\theta} \leq U\left(\Psi_{m}\right),
\end{aligned}
$$

where:

$$
\begin{aligned}
\bar{\Psi}_{m} & =\psi_{m}\left(\frac{\psi_{m}}{27 \psi_{\theta}}-1\right) \\
L\left(\Psi_{m}\right) & =\psi_{\theta} B^{(1)}\left(\frac{\Psi_{m}}{\psi_{m}}\right) \\
U\left(\Psi_{m}\right) & =\psi_{\theta} B^{(2)}\left(\frac{\Psi_{m}}{\psi_{m}}\right) .
\end{aligned}
$$

Note that $L\left(\Psi_{m}\right)$ and $U\left(\Psi_{m}\right)$ are implicit functions of $\left(\psi_{m}, \psi_{\theta}\right)$. By (R7), the boundaries $L\left(\Psi_{m}\right)$ and $U\left(\Psi_{m}\right)$ are decreasing functions of $\Psi_{m}$. Also, because $a_{2}\left(\rho_{m}\right)$ is decreasing, it follows from (42) that $B^{(2)}\left(\rho_{m}\right)$ is convex. Lastly, by $(\mathrm{R} 6)$, we have that $L\left(\bar{\Psi}_{m}\right)=U\left(\bar{\Psi}_{m}\right)$.

Now the first two points of Proposition 4 follow from (R8)-(R12). The last two points follow from an application of the IFT on the boundaries. Namely, on the upper boundary, one applies the IFT at $A=A_{L}$, because $\partial G / \partial A\left(A_{L}\right)<0$. This shows that the unique equilibrium above the boundary converges towards $A_{L}$ as $\left(\Psi_{m}, \Psi_{\theta}\right)$ approaches the boundary from above. Note that the reasoning does not apply to the other equilibrium, $A_{H}$ : indeed, because $\partial G / \partial A\left(A_{H}\right)=0$, one cannot apply the IFT at $A=A_{H}$. Similarly, on the lower boundary, one applies the IFT to $A=A_{H}$, but cannot apply it at $A=A_{L}$ because $\partial G / \partial\left(A_{L}\right)=0$.

\section{A.3 Proof of Proposition 5}

The ex ante time- $t$ flow welfare of a household is

$$
\mathbb{E}_{0}\left[\log C_{i t}-\Theta L_{i t}\right]=\mathbb{E}_{0}\left[\mathbb{E}_{i t}\left[\log C_{i t}\right]-\mathbb{E}_{i t}[\Theta] L_{i t}\right]=-\mathbb{E}_{0} \log \mathbb{E}_{i t}[\Theta]+\log \beta-\beta
$$

where we used that $C_{i t}=L_{i t}=\beta \mathbb{E}_{i t}[\Theta]^{-1}$ together with the law of iterated expectations. From period 2 onwards, $\mathbb{E}_{i t} \Theta=\Theta$. And we know that $\mathbb{E}_{0}[\log \Theta]=0$ by the prior distribution assumption. So, $\mathbb{E}_{0} \log \mathbb{E}_{i t}[\Theta]=\mathbb{E}_{0}[\log \Theta]=0$. In the first period, we have that 
$\mathbb{E}_{0} \log \mathbb{E}_{i 1}[\Theta]=\mathbb{E}_{0}\left[\mathbb{E}_{i 1} \log \Theta\right]+\operatorname{var}_{i 1}[\log \Theta] / 2$. Using the law of iterated expectations and that $\operatorname{var}_{i 1}[\log \Theta]=\left(\psi_{\theta}+A^{2} \psi_{m}+\Psi_{\theta}+A^{2} \Psi_{m}\right)^{-1}$, and adding up through time, the result follows.

\section{A.4 Proof of Lemma 4}

We will be using the notations and functions defined in the proof of Appendix A.2. We first

note at the highest equilibrium $A$, the best reply $H\left(\psi_{\theta}+A^{2} \psi_{m}, \Psi_{\theta}+A^{2} \Psi_{m}\right)$ must cross the 45-degree line from above. Since, at the same time, the best reply decreases in both $\left(\Psi_{\theta}, \Psi_{m}\right)$, the result follows. Continuity follows from the IFT, which we can apply everywhere except on the upper boundary $\Psi_{\theta}=U\left(\Psi_{m}\right)$, because $\partial G / \partial A\left(A_{H}, \rho_{m}\right)=0$.

Fix $\Psi_{\theta}$ and let $\Psi_{m}$ go to infinity. Given that $A_{\star}$ decreases in $\Psi_{m}$ and is bounded below by $0, A_{\star}$ converges to a finite limit as $\Psi_{m}$ tends to infinity. Clearly, this limit cannot be positive, or else equation (31) cannot be satisfied for sufficiently high $\Psi_{m}$. Hence $A_{\star}$ tends to 0 as $\Psi_{m}$ tends to infinity.

From the equilibrium equation (31), it follows that

$$
0 \leq A_{\star}=H\left(\psi_{\theta}+A_{\star}^{2} \psi_{m}, \Psi_{\theta}+A_{\star}^{2} \Psi_{m}\right) \leq H\left(\psi_{\theta}+\psi_{m}, \Psi_{\theta}\right)=\frac{\psi_{\theta}+\psi_{m}}{\psi_{\theta}+\psi_{m}+\Psi_{\theta}} .
$$

The first inequality follows from the fact that $H\left(\psi_{\theta}^{\prime}, \Psi_{\theta}^{\prime}\right)$ is increasing in $\psi_{\theta}^{\prime}$ and decreasing in $\Psi_{\theta}^{\prime}$, together with the observation that $A_{\star} \in[0,1]$. Now, letting $\Psi_{\theta}$ go to infinity, one sees that $A_{\star}$ must go to zero.

\section{A.5 Proof of Proposition 6}

We will be using the notations and the functions defined in the proof of Appendix A.2. First, recall that, using the fixed-point equation, the posterior precision can be written

$$
\Pi=\psi_{\theta}+\Psi_{\theta}+A_{\star}^{2} \psi_{m}+A_{\star}^{2} \Psi_{m}=\frac{\psi_{\theta}}{A_{\star}}+A_{\star} \psi_{m} .
$$

Taking derivatives with respect to $A_{\star}$, one finds that

$$
\frac{\partial \Pi}{\partial A_{\star}}=\frac{-\psi_{\theta}+A_{\star}^{2} \psi_{m}}{A_{\star}^{2}},
$$

Now recall that $A_{\star}$ is decreasing in both $\Psi_{m}$ and $\Psi_{\theta}$. By proposition 4, along an increasing path of $\Psi_{m}$ and $\Psi_{\theta}, A_{\star}$ is continuous except at the upper frontier where it jumps down. It 
then follows that,

(R13) If $A_{\star}$ is continuous, then a marginal increase in public information reduces welfare if and only if $A_{\star}>\sqrt{1 / \lambda}$.

Where, keeping the notations of the previous proof, $\lambda=\psi_{m} / \psi_{\theta}$. Now we show:

(R14) Suppose there is a unique equilibrium. Then, a marginal increase in public information decreases welfare if and only if

$$
\rho_{\theta}<2(\sqrt{\lambda}-1)-\rho_{m} \equiv C\left(\rho_{m}\right)
$$

Indeed, recall that if there is a unique equilibrium, $A_{\star}$ is continuous, and the best reply is above the 45-degree line for all $A<A_{\star}$ and below the 45-degree line for all $A>A_{\star}$. Thus, $\sqrt{1 / \lambda}<A_{\star}$ if and only if, when $A=\sqrt{1 / \lambda}$, the best reply is above the 45 -degree line. Plugging $A=\sqrt{1 / \lambda}$ in the inequality $H\left(\psi_{\theta}+A^{2} \psi_{m}, \Psi_{\theta}+A^{2} \Psi_{m}\right)>A$ and rearranging, one obtains the condition (R14).

Now consider parameters $\lambda$ and $\Psi_{m}$ such that there are multiple equilibria. We start by showing:

(R15) Suppose $\lambda>27$ and $\rho_{m}<\sqrt{\lambda / 27}-1$. Then, $a_{1}>\sqrt{1 / \lambda}$.

Indeed, note that, if $A \leq \sqrt{1 / \lambda}$, then $D\left(A, \rho_{m}\right)<0$. Since $D\left(a_{1}, \rho_{m}\right)=0$, it thus follows that $a_{1}>\sqrt{1 / \lambda}$. Next, we prove:

(R16) Suppose $\lambda>27$ and $\rho_{m}<\sqrt{\lambda / 27}-1$. Then, for all $\rho_{\theta}<B^{(2)}\left(\rho_{m}\right)$, welfare decreases continuously with public information.

Indeed, first recall from $(\mathrm{R} 8)$ that for all $\rho_{\theta}<B^{(1)}\left(\rho_{m}\right)$, there is a unique equilibrium, $A_{\star}$, that is larger than $a_{2}$, and thus larger than $a_{1}$. Then, for all $B^{(1)}\left(\rho_{m}\right) \leq \rho_{\theta}<B^{(2)}\left(\rho_{m}\right)$, the largest equilibrium, $A_{\star}$, is also larger than $a_{1}$. Because of (R15), it follows that for all $\rho_{\theta}<B^{(2)}\left(\rho_{m}\right), A_{\star}>\sqrt{1 / \lambda}$. Thus, from (R13), we know that, for all $\rho_{\theta}<B^{(2)}\left(\rho_{m}\right)$, a marginal increase in public information reduces welfare.

Taken together, (R14) and (R16), we can generalize to:

(R17) Welfare decreases continuously with public information if and only if

$$
\begin{array}{cl}
\rho_{\theta}<\max \left\{B^{(2)}\left(\rho_{m}\right), C\left(\rho_{m}\right)\right\} & \text { whenever } B^{(2)}\left(\rho_{m}\right) \text { is defined } \\
\rho_{\theta}<C\left(\rho_{m}\right) & \text { otherwise. }
\end{array}
$$


Welfare jumps down with public information if and only if $\rho_{\theta}=B^{(2)}\left(\rho_{m}\right)$.

Indeed if $B^{(2)}\left(\rho_{m}\right)$ is not defined then this is the same as (R14). If $B^{(2)}\left(\rho_{m}\right)$ is defined, let's start with the "only if" part. If public information decreases welfare, then either (i) $\rho_{\theta}<B^{(2)}\left(\rho_{m}\right)$ or (ii) $\rho_{\theta}>B^{(2)}\left(\rho_{m}\right)$, the equilibrium is unique and by $(\mathrm{R} 14), \rho_{\theta}<$ $C\left(\rho_{m}\right)$. Either way, $\rho_{\theta}<\max \left\{B^{(2)}\left(\rho_{m}\right), C\left(\rho_{m}\right)\right\}$. For the "if" part, suppose that $\rho_{\theta}<$ $\max \left\{B^{(2)}\left(\rho_{m}\right), C\left(\rho_{m}\right)\right\}$. Then, either $\rho_{\theta} \leq B^{(2)}\left(\rho_{m}\right)$ and by (R17) a marginal increase in public information decreases welfare. Or $\rho_{\theta}>B^{(2)}\left(\rho_{m}\right)$ and $\rho_{\theta}<C\left(\rho_{m}\right)$, the equilibrium is unique, and a marginal increase in public information decreases welfare. The last point follows directly from the fact that, when $\rho_{\theta}=B^{(2)}\left(\rho_{m}\right)$, the aggregate weight $A_{\star}$ jumps down.

The result of the Proposition 6 then follows directly by plugging in the above $\rho_{\theta}=\Psi_{\theta} / \psi_{\theta}$, $\rho_{m}=\Psi_{m} / \psi_{m}$ and $\lambda=\psi_{m} / \psi_{\theta}$. The following additional result further characterizes the shape of the boundary, showing among other things that $U\left(\bar{\Psi}_{m}\right) \leq \Delta\left(\bar{\Psi}_{m}\right)$.

\section{An Additional Result}

We now characterize further the shape of the boundary. Namely, we show

Lemma 7. Suppose that $0 \leq \rho_{m}<\sqrt{\lambda / 27}-1$. Then, $B^{(2)}\left(\rho_{m}\right)<C\left(\rho_{m}\right)$ if and only if

$$
\rho_{m}>\max \{0, \sqrt{\lambda}(3-2 \sqrt{2})-1\}
$$

Note that $\sqrt{\lambda}(3-2 \sqrt{2})-1$ is less than $\sqrt{\lambda / 27}-1 ;$ thus, when $\rho_{m}=\sqrt{\lambda / 27}-1, C\left(\rho_{m}\right)>$ $B^{(2)}\left(\rho_{m}\right)$.

In words, the lemma says that $B^{(2)}\left(\rho_{m}\right)$ is above $C\left(\rho_{m}\right)$ when $\rho_{m}$ is less than $\max \{0, \sqrt{\lambda}(3-$ $2 \sqrt{2})-1\}$ and below $C\left(\rho_{m}\right)$ after. So the boundary of result (R17) coincides with $B^{(2)}\left(\rho_{m}\right)$ for low values of $\rho_{m}$, and with $C\left(\rho_{m}\right)$ for high values. Also, it can be that $\lambda>27$ and $\sqrt{\lambda}(3-2 \sqrt{2})-1<0$; in that case, $C\left(\rho_{m}\right)>B^{(2)}\left(\rho_{m}\right)$ all along. We now proceed to prove the lemma, starting with

(R18) Suppose that $0 \leq \rho_{m}<\sqrt{\lambda / 27}-1$. Then, $B^{(2)}\left(\rho_{m}\right)<C\left(\rho_{m}\right)$ if and only if, when $\rho_{\theta}=C\left(\rho_{m}\right)$, there is a unique equilibrium.

Consider first the "only if" part. Suppose that $B^{(2)}\left(\rho_{m}\right)<C\left(\rho_{m}\right)$ and let $\rho_{\theta}=C\left(\rho_{m}\right)$. Then, $\rho_{\theta}>B^{(2)}\left(\rho_{m}\right)$ so there is a unique equilibrium by (R12). Thus, there is a unique 
equilibrium. We proceed with the "if" part. Suppose that, when $\rho_{\theta}=C\left(\rho_{m}\right)$, there is a unique equilibrium. Thus, $\left(\rho_{m}, \rho_{\theta}\right)$ could be either above the upper boundary, $\rho_{\theta}>B^{(2)}\left(\rho_{m}\right)$, or below the lower boundary, $\rho_{\theta}<B^{(1)}\left(\rho_{m}\right)$. But, if $\left(\rho_{m}, \rho_{\theta}\right)$ were below the lower boundary, then, by (R16), an increase in $\rho_{\theta}$ would decrease welfare, which is impossible because $\rho_{\theta}=$ $C\left(\rho_{m}\right)$.

Now consider the equilibrium equation $G\left(A, \rho_{m}\right)=\rho_{\theta}$, when $\rho_{\theta}=C\left(\rho_{m}\right)$. Straightforward algebraic manipulation shows that it can be written:

$$
\lambda\left(A^{3}\left(1+\rho_{m}\right)-A^{2}\right)+A\left(2 \sqrt{\lambda}-\left(1+\rho_{m}\right)\right)-1=0
$$

One sees that $A=\sqrt{1 / \lambda}$ is a solution of this equation, so that it can be factorized:

$$
(A \sqrt{\lambda}-1)\left[A^{2}\left(1+\rho_{m}\right) \sqrt{\lambda}+A\left(1+\rho_{m}-\sqrt{\lambda}\right)+1\right]=0
$$

Clearly, $A=\sqrt{\lambda}$ is the unique solution if and only if the discriminant of the second multiplicative term is negative, i.e., if and only if

$$
\left(1+\rho_{m}\right)^{2}-6\left(1+\rho_{m}\right) \sqrt{\lambda}+\lambda<0 .
$$

This second-order polynomial has two roots:

$$
\begin{aligned}
& \rho_{m}=\sqrt{\lambda}(3-2 \sqrt{2})-1 \\
& \rho_{m}=\sqrt{\lambda}(3+2 \sqrt{2})-1
\end{aligned}
$$

The first root is smaller than $\sqrt{\lambda / 27}-1$; the second root is greater. Note that the first root may be less than zero. It then follows that, when $0 \leq \rho_{m}<\sqrt{\lambda / 27}-1$, the discriminant is negative if and only if $\rho_{m}>\max \{0, \sqrt{\lambda}(3-2 \sqrt{2})-1\}$, which, together with (R18), proves the lemma.

\section{A.6 Proof of Corollary 1}

Consider three vectors $\Psi^{1}=\left(\Psi_{m}^{(1)}, \Psi_{\theta}^{(1)}\right), \Psi^{(2)}=\left(\Psi_{m}^{(2)}, \Psi_{\theta}^{(2)}\right)$ and $\Psi_{\theta}^{(3)}=\left(\Psi_{m}^{(3)}, \Psi_{\theta}^{(3)}\right)$ such that

$$
\begin{aligned}
& \Psi_{m}^{(1)} \leq \Psi_{m}^{(2)} \leq \Psi_{m}^{(3)} \\
& \Psi_{\theta}^{(1)} \leq \Psi_{\theta}^{(2)} \leq \Psi_{\theta}^{(3)},
\end{aligned}
$$


and let $W\left(\Psi^{(i)}\right)$ be the level of utilitarian welfare associated with $\Psi^{(i)}$. Now, there are only two possibilities: If $\Psi^{(2)}$ lies below the boundary $\Psi_{\theta}=\Gamma\left(\Psi_{m}\right)$, so does $\Psi^{(1)}$ and it follows that $W\left(\Psi^{(2)}\right) \leq W\left(\Psi^{(1)}\right)$. If, on the other hand, $\Psi^{(2)}$ lies above the boundary $\Psi_{\theta}=\Gamma\left(\Psi_{m}\right)$, then so does $\Psi^{(3)}$, and $W\left(\Psi^{(2)}\right) \leq W\left(\Psi^{(3)}\right)$. Taken together, we obtain

$$
W\left(\Psi^{(2)}\right) \leq \max \left\{W\left(\Psi^{(1)}\right), W\left(\Psi^{(3)}\right)\right\}
$$

Now suppose the government has information that would allow an increase in the exogenous public knowledge from $\Psi^{(1)}$ up to $\Psi^{(3)}$. The last inequality shows that any intermediate announcement, $\Psi^{(2)}$, will always be dominated by either saying nothing at all, $\Psi^{(1)}$, or releasing all the information, $\Psi^{(3)}$.

\section{A.7 Proof of Proposition 7}

As shown in the text, the posterior precision can be written:

$$
\frac{\psi_{\theta}}{A_{\star}}+\psi_{m} A_{\star}
$$

As $\Psi_{m}$ or $\Psi_{\theta}$ go to infinity, $A_{\star}$ goes to zero and so posterior precision goes to infinity. Thus, a sufficiently large increase in $\Psi_{m}$ or $\Psi_{\theta}$ will increase posterior precision, and thus welfare. The second point of the proposition is shown in the text.

\section{A.8 Proof of Propositions 8 to 10}

Let us define $A \equiv a_{1} / a_{2}$ and $B \equiv a_{1} / a_{3}$ and $C \equiv a_{1} /\left(a_{4}-1\right)$. The observing the price plus noise is equivalent to observing

$$
\hat{p}=\theta+m / A+v / B+\varepsilon_{p} / C
$$

The household observes then $\hat{p}$ together with $\theta_{i}, m_{i}$ and $v_{i}$. One can then show that the household's posterior about $\theta$ is normal with a mean

$$
\mathbb{E}_{i}[\theta]=\frac{\psi_{\theta}}{\kappa} \theta_{i}+\frac{\kappa-\psi_{\theta}-\Psi_{\theta}}{\kappa}\left(\hat{p}-\frac{\psi_{m}}{\psi_{m}+\Psi_{m}} \frac{m_{i}}{A}-\frac{\psi_{v}}{\psi_{v}+\Psi_{v}} \frac{v_{i}}{B}\right)
$$


and precision $\kappa$ given by

$$
\kappa=\psi_{\theta}+\Psi_{\theta}+\left[\frac{1}{A^{2}\left(\psi_{m}+\Psi_{m}\right)}+\frac{1}{B^{2}\left(\psi_{v}+\Psi_{v}\right)}+\frac{1}{C^{2} \Psi_{p}}\right]^{-1}
$$

Now, aggregating across consumers and ignoring all terms that are common knowledge, we obtain that

$$
\begin{aligned}
p+\varepsilon_{p} & =\int \mathbb{E}_{i}[\theta] d i+m+v+\varepsilon_{p} \\
& =\frac{\psi_{\theta}}{\kappa} \theta+\left[1-\frac{\left(\kappa-\psi_{\theta}-\Psi_{\theta}\right) \psi_{m} / A}{\left(\psi_{m}+\Psi_{m}\right) \kappa}\right] m+\left[1-\frac{\left(\kappa-\psi_{\theta}-\Psi_{\theta}\right) \psi_{v} / B}{\left(\psi_{v}+\Psi_{v}\right) \kappa}\right]+\varepsilon_{p} .
\end{aligned}
$$

After identifying the unknown coefficient $A, B$, and $C$, we obtain:

$$
\begin{aligned}
& \frac{1}{A}=\kappa\left[\psi_{\theta}+\frac{\left(\kappa-\psi_{\theta}-\Psi_{\theta}\right) \psi_{m}}{\psi_{m}+\Psi_{m}}\right]^{-1} \\
& \frac{1}{B}=\kappa\left[\psi_{\theta}+\frac{\left(\kappa-\psi_{\theta}-\Psi_{\theta}\right) \psi_{v}}{\psi_{v}+\Psi_{v}}\right]^{-1} \\
& \frac{1}{C}=\frac{\kappa}{\psi_{\theta}}
\end{aligned}
$$

Let us define $G(\kappa)$ to be the right hand side of equation 43 after substituting in for the above values of $A, B$, and $C$. Existence of an equilibrium follows by noticing that $G\left(\psi_{\theta}+\Psi_{\theta}\right)>\psi_{\theta}+\Psi_{\theta}$ and $\lim _{\kappa \rightarrow \infty} G(\kappa)<\infty$. This finishes the proof of the result in proposition 8 .

In order to prove Proposition 9, we first take derivative of $G(\kappa)$ with respect to $\Psi_{i}$. We obtain that, for a given $\kappa$, the function $G(\kappa)$ decreases with a marginal increase of $\Psi_{i}$ only if

$$
\frac{\Psi_{i}}{\psi_{i}}<\frac{\kappa_{\star}-\Psi_{\theta}}{\psi_{\theta}}-2
$$

Since, at $\kappa=\kappa_{\star}$, the function $G(\kappa)$ crosses the 45 degree line from above, it follows that if the above inequality is satisfied, a marginal increase in $\Psi_{i}$ lowers $\kappa_{\star}$, and vice versa if the reverse inequality is satisfied.

Finally to proof Proposition 10, note that if $\Psi_{m}=\infty$, then the fixed point equation converges to

$$
\kappa=\bar{G}(\kappa)=\psi_{\theta}+\Psi_{\theta}+\left[\frac{1}{B^{2}\left(\psi_{v}+\Psi_{v}\right)}+\frac{1}{C^{2} \Psi_{p}}\right]^{-1}
$$


Note that $\bar{G}(\kappa)>G(\kappa)$ for $\kappa>\psi_{\theta}+\Psi_{\theta}$, implying that the highest fixed point of $\bar{G}(\kappa)$ is larger than the highest fixed point of $G(\kappa)$. A similar argument applies to $\Psi_{v}=\infty$. The case of $\Psi_{p}=\infty$ follows more directly as marginal increases are always beneficial in this case. 


\section{References}

Amador, Manuel and Pierre-Olivier Weill, "Learning from Private and Public Observations of Others' Actions," 2006. Working Paper, Stanford University and UCLA. 5, 26

Angeletos, George-Marios and Alessandro Pavan, "Policy with Dispersed Information," 2007. Working Paper, MIT and Northwestern University. 6

- and Ivan Werning, "Crises and Prices: Information Aggregation, Multiplicity and Volatility," American Economic Review, December 2006, 96, 1721-1737. 28

Atkeson, Andrew, V.V. Chari, and Patrick Kehoe, "On the Optimal Choice of a Monetary Policy Instrument," 2007. Working paper, Federal Reserve Bank of Minneapolis. 6

Bils, Marc and Peter Klenow, "Some Evidence on the Importance of Sticky Prices," Journal of Political Economy, 2004, 112, 947-985. 4

Brunermeier, Markus K., Asset Pricing under Asymmetric Information, Oxford Univeristy Press, 2001. 22

Constantinides, George and Darrell Duffie, "Asset Pricing with Heterogenous Consumers," Journal of Political Economy, 1996, 104, 219-240. 34

Eusepi, Stefano and Bruce Preston, "Central Bank Communication and Expectations Stabilization," 2007. Working Paper, Department of Economics, Columbia University. 6

Ganguli, Jayant V. and Lyian Yang, "Complementarities and Multiplicity with a Common Endowment Shock," 2007. Working Paper, Cornell University. 28

Grossman, Sanford J., "On the Efficiency of Competitive Stock Markets Where Trades Have Diverse Information," Journal of Finance, 1975, 31, 573-585. 5, 12

Hellwig, Christian, "Heterogeneous Information and the Welfare Effects of Public Information Disclosures," 2005. 6

_, Arijit Mukherji, and Aleh Tsyvinski, "Self-Fulfilling Currency Crises: The Role of Interest Rates," American Economic Review, December 2006, 96, 1769-1787. 28 
Hellwig, Martin, "On the aggregation of information in competitive markets," Journal of Economic Theory, 1980, 22, 477-498. 5, 12

Hirshleifer, Jack, "The private and social value of information and the reward to incentive activity," American Economic Review, 1971, 61, 561-574. 22

Lorenzoni, Guido, "New Shocks and Optimal Monetary Policy," 2007. Working Paper, MIT. 6

Lucas, Robert E. Jr., "Expectations and the Neutrality of Money," Journal of Economic Theory, 1972, 4, 103-124. 2, 5

Morris, Stephen and Hyun Song Shin, "The Social Value of Public Information," American Economic Review, 2002, 92, 1521-1534. 5, 6, 27, 28

Moscarini, Guiseppe, "Competence Implies Credibility," American Economic Review, 2007, 97, 37-63. 6

Roca, Mauro, "Transparency and Monetary Policy with Imperfect Common Knowledge," 2006. Working Paper, Columbia University. 6

Sun, Yeneng, "The exact law of large numbers via Fubini extension and characterization of insurable risks," Journal of Economic Theory, 2006, 126, 31-69. 9

Svensson, Lars, "Social Value of Public Information: Morris and Shin (2002) Is Actually Pro Transparency, Not Con,", American Economic Review, 2006, 96, 448-451. 28

Taub, Bart, "Optimal Policy in a Model of Endogenous Fluctuations and Assets," Journal of Economic Dynamics and Control, 1997, 21. 6

Townsend, Robert M., "Forecasting the Forecasts of Others," Journal of Political Economy, 1983, 91, 546-588. 7 